\title{
Effectiveness of different adjunctive interventions in the management of orthodontically induced white spot lesions: A systematic review of systematic reviews and meta-analyses
}

\section{Skuteczność różnych metod leczenia poortodontycznych białych plam próchnicowych - przegląd systematyczny z przeglądów systematycznych i metaanaliz}

\author{
Wesam Mhd Mounir Bakdach ${ }^{B-D, F}$, Rania Hadad ${ }^{A, E, F}$ \\ Department of Orthodontics, Faculty of Dentistry, University of Damascus, Syria \\ A - research concept and design; B - collection and/or assembly of data; $\mathrm{C}$ - data analysis and interpretation; \\ $\mathrm{D}$ - writing the article; $\mathrm{E}$ - critical revision of the article; $\mathrm{F}$ - final approval of the article
}

Address for correspondence

Wesam Mhd Mounir Bakdach

E-mail: bakdach-wm@hotmail.com

Funding sources

None declared

Conflict of interest

None declared

Acknowledgements

The authors would like to thank Dr. Tuqa Raghis for generously

sharing the clinical photograph provided in Fig. 2.

Received on December 6, 2019

Reviewed on February 5, 2020

Accepted on February 20, 2020

Published online on September 30, 2020

Cite as

Bakdach WMM, Hadad R. Effectiveness of different adjunctive interventions in the management of orthodontically induced white spot lesions: A systematic review of systematic reviews and meta-analyses. Dent Med Probl. 2020;57(3):305-325. doi:10.17219/dmp/118330

DOI

10.17219/dmp/118330

Copyright

(c) 2020 by Wroclaw Medical University

This is an article distributed under the terms of the

Creative Commons Attribution 3.0 Unported License (CC BY 3.0)

(https://creativecommons.org/licenses/by/3.0/).

\begin{abstract}
The purposes of this review were to appraise the current evidence on the management of orthodontically induced white spot lesions (OIWSLs) and to choose the best evidence from among conflicting systematic reviews.

The published literature was searched from inception through November 2019 in 5 databases. Only systematic reviews and/or meta-analyses were eligible for inclusion. Methodological quality was assessed using A Measurement Tool to Assess Systematic Reviews-2 (AMSTAR-2). The Jadad decision algorithm was applied to choose the best available evidence from among discordant reviews.

Thirteen publications were included. The interventions reported in the management of OIWSLs were topical fluorides, casein phosphopeptide-amorphous calcium phosphate (CPP-ACP)-containing products, fluoride-containing bonding materials, laser therapy, resin infiltration, and micro-abrasion. The methodological quality of the reviews ranged between moderate and critically low according to the AMSTAR-2 tool. Based on the Jadad decision algorithm criteria, topical fluorides yielded a $25-30 \%$ prevention of OIWSLs; however, their effect on reversing OIWSLS was unclear. The CPP-ACP products were effective in both preventing and reversing OIWSLS. No differences were noted between fluoride-releasing adhesives and conventional adhesives. Laser irradiation was effective in preventing OIWSLS, with some concerns about the argon laser at a certain setting. Finally, there is a lack of reliable evidence supporting the efficacy of resin infiltration or micro-abrasion due to the limited number of available studies.
\end{abstract}

Based on the currently available information, topical fluorides and laser irradiation are effective in preventing OIWSLS. The CPP-ACP products are effective in preventing and reversing OIWSLS. Fluoride-releasing adhesives have no effect on OIWSL prevention.

Key words: orthodontics, dental care, tooth demineralization, tooth remineralization, white caries spot Słowa kluczowe: ortodoncja, opieka stomatologiczna, demineralizacja zębów, remineralizacja zębów, biała plama próchnicowa 


\section{Introduction}

Achieving optimal occlusion with minimal treatment complications is an essential demand for orthodontic treatment. ${ }^{1}$ Orthodontically induced white spot lesions (OIWSLs) are considered one of the most prominent complications, with a prevalence of $68.4 \%$ in patients undergoing multi-bracketed fixed orthodontic treatment. ${ }^{2}$ This high percentage of prevalence is due to the irregular and non-uniform surfaces of fixed appliances, which can encourage plaque stagnation and limit natural self-cleansing mechanisms (Fig. 1,2). ${ }^{3-5}$

Primarily, patients seek orthodontic treatment in an attempt to improve function and esthetics. ${ }^{4}$ However, these common white, chalky, opaque spots with limited

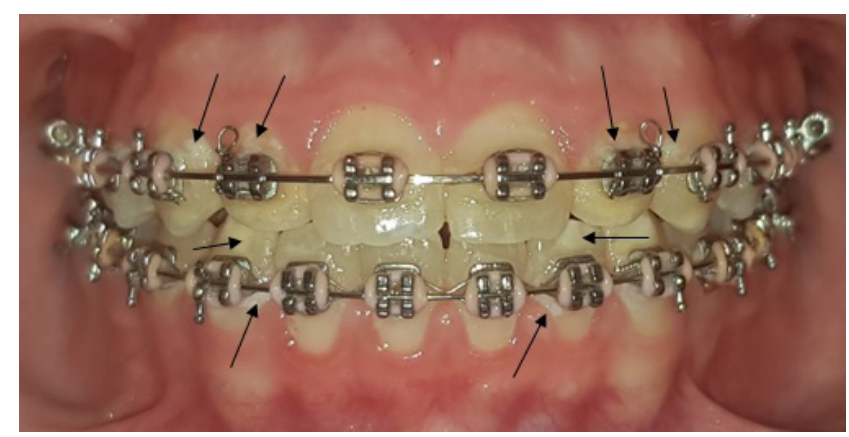

Fig. 1. Clinical case undergoing multi-bracketed fixed orthodontic treatment and developing several orthodontically induced white spot lesions (OIWSLs)

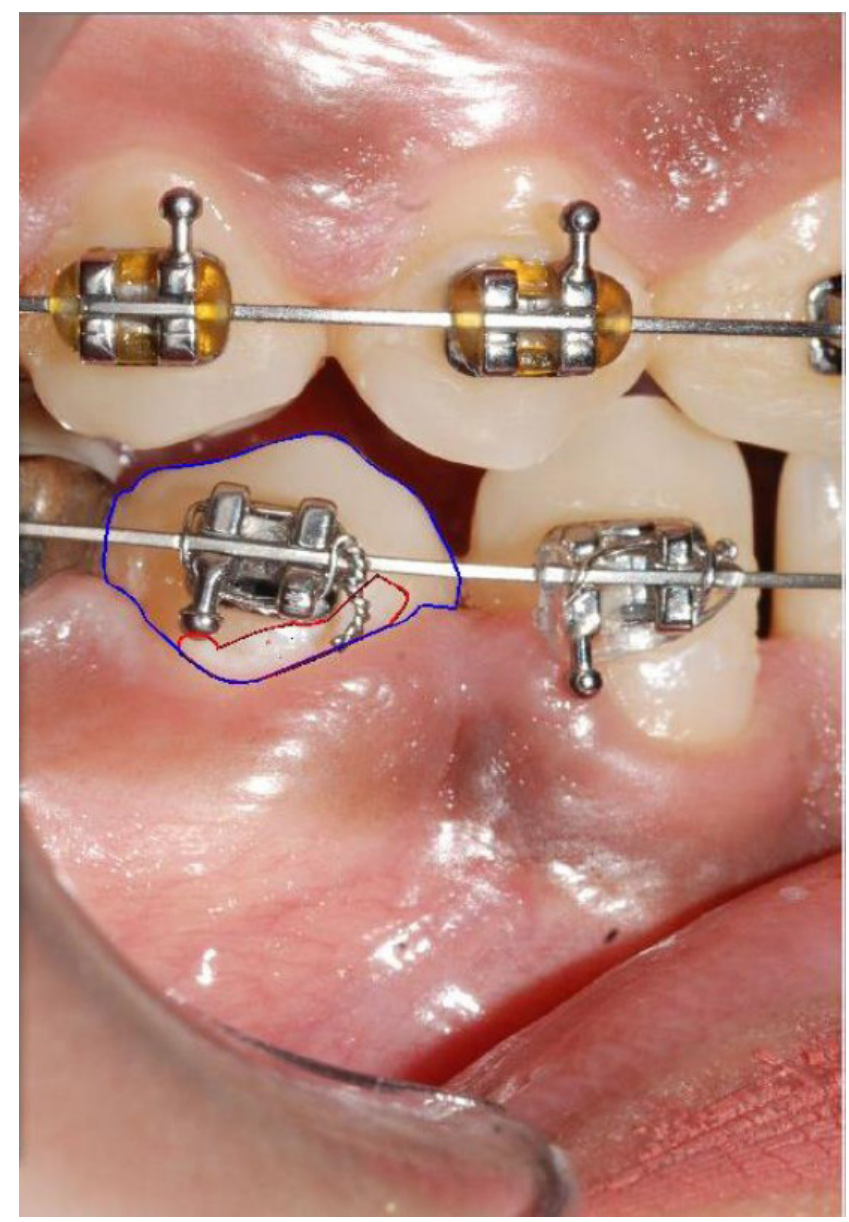

Fig. 2. Orthodontically induced white spot lesion (OIWSL) spontaneous reversibility may seriously jeopardize esthetic appearance and the patient's satisfaction with the results of treatment. ${ }^{4,6,7}$ Worse still, untreated white spot lesions (WSLs) may lead to tooth cavitation, requiring a subsequent restorative procedure. ${ }^{8}$

It is important to differentiate OIWSLs from other, non-carious white spots, such as fluorosis, developmental enamel hypomineralization or enamel hypoplasia. Firstly, OIWSLs are typically found on the buccal tooth surfaces and around the perimeter of orthodontic brackets whereas non-carious spots usually cover the entire tooth surface and are not associated with orthodontic treatment. ${ }^{9,10}$ Secondly, the consistency and texture of the surface of a clean, dry tooth can be gently assessed with a periodontal probe. Orthodontically induced white spot lesions appear rough, opaque and porous, while noncarious lesions appear mostly smooth and shiny. ${ }^{10}$

Numerous research projects have been conducted on the effectiveness of various interventions in the management of OIWSLs. Mainly, the approaches involved either preventing the formation of lesions or reversing the existing ones through 2 basic strategies - remineralizing the enamel or masking the lesions. ${ }^{7}$

Recently, exponential growth in the systematic reviews focusing on this issue has been noticed. However, given the breadth of this topic area, decision-makers have become overwhelmed by a plethora of reviews reporting contradictory conclusions. Thus, a systematic review of the published reviews (an overview or umbrella review) was a logical and appropriate next step, in order to summarize the body of evidence and choose from among the disparate findings of various reviews.

\section{Methods}

\section{Protocol and registration}

The methodology of this review was formulated in advance by strictly adhering to the Preferred Reporting Items for Systematic Reviews and Meta-Analyses (PRISMA) standards. The systematic review protocol was written during the first phase of this review and registered in the International Prospective Register of Systematic Reviews (PROSPERO) under the number CRD42019135137, which is freely available at https://www.crd.york.ac.uk/ prospero/display_record.php?RecordID=135137.

\section{Question}

The research question with regard to the effectiveness of different adjunctive interventions in the management of OIWSLs was defined according to the PICO format: - P (population) - healthy patients of any age and from any ethnic group who underwent multi-bracketed fixed orthodontic treatment; 
- I (intervention) - any adjunctive intervention that would prevent or reverse (manage) OIWSLs;

- C (comparison) - a control group that was not exposed to an adjunctive intervention;

- O (outcome) - any measurement that would reflect the efficacy of the applied intervention, such as changes in the incidence or size of the lesions, or any quantitative assessment of enamel mineral loss.

\section{Eligibility criteria}

The following criteria were used to consider articles eligible for this review:

- only systematic reviews and/or meta-analyses were eligible for inclusion;

- only interventions that involve remineralizing the enamel or masking the lesions were included;

- due to the scarcity of randomized controlled trials (RCTs) on this subject, the design of the primary studies of the eligible reviews were either controlled clinical trials (CCTs) (randomized or non-randomized) or prospective observational studies with concurrent comparison.

Trials, narrative literature reviews, reviews including the methods of improving patients' oral hygiene, reviews including laboratory primary studies, and reviews discussing the management of naturally developed WSLs (i.e., without orthodontic treatment) were excluded.

\section{Search strategy}

A comprehensive electronic search was performed from inception through November 2019 in the following databases: Cochrane Library, Scopus, Web of Science,
CINAHL via EBSCO, and PubMed. Additionally, the bibliographies of the relevant reviews were screened for further possible reviews. No restrictions were applied on the search strategy with regard to the language, the publication date or the publication status. More details on the search strategy can be found in Supplementary Table 1.

\section{Study selection and data extraction}

The authors of the present study independently assessed the identified results of the search and extracted necessary data. The screening process was initiated by assessing titles and abstracts. Then, the full text of each relevant article was evaluated in depth. Articles that failed to meet one or more of the eligibility criteria were eliminated. After filtration, the required data was extracted from the studies using a standardized form that included the authors' names, setting, the number of primary studies, interventions, the number of participants, outcomes, the quality of primary studies, and main findings. Any disagreement between the 2 reviewers was discussed until a resolution was reached.

\section{Quality assessment of the selected reviews}

The methodological quality of the obtained systematic reviews was assessed independently by the 2 reviewers utilizing A Measurement Tool to Assess Systematic Reviews-2 (AMSTAR-2). ${ }^{11}$ The AMSTAR-2 checklist includes 16 domains that assess the multiple steps of conducting a systematic review, of which 7 domains are considered critical.

Supplementary Table 1. Search strategy

\begin{tabular}{|c|c|c|}
\hline Database & Search strategy & Results \\
\hline $\begin{array}{l}\text { Cochrane Library } \\
\text { from inception up to November 30, } 2019 \\
\text { Title Abstract Key Word } \\
\text { with no limits }\end{array}$ & $\begin{array}{l}\text { \#1 orthodontic* OR "Orthodontic treatment" } \\
\text { \#2 "White lesions" OR "White spot lesions" OR "White spots" OR demineraliz* OR decalcif* OR remineraliz* } \\
\text { \#3 manag* OR reduc* OR prevent* OR revers* } \\
\text { \#4 "systematic review" OR "meta-analysis" } \\
\text { \#5 \#1 AND \#2 AND \#3 AND \#4 }\end{array}$ & $\begin{array}{c}\# 5=33 \\
\text { (reviews) }\end{array}$ \\
\hline $\begin{array}{l}\text { Scopus } \\
\text { from inception up to November 30, } 2019 \\
\text { Title Abstract Key Word } \\
\text { with no limits }\end{array}$ & $\begin{array}{l}\text { \#1 orthodontic* OR "orthodontic treatment" } \\
\text { \#2 "White lesions" OR "White spot lesions" OR "White spots" OR demineraliz* OR decalcif* OR remineraliz* } \\
\text { \#3 manag* OR reduc* OR prevent* OR revers* } \\
\text { \#4 "systematic review" OR "meta-analysis" } \\
\text { \#5 \#1 AND \#2 AND \#3 AND \#4 }\end{array}$ & $\# 5=33$ \\
\hline $\begin{array}{l}\text { Web of Science } \\
\text { from inception up to November 30, } 2019 \\
\text { TS - Topic } \\
\text { with no limits }\end{array}$ & $\begin{array}{l}\text { \#1 TS }=(\text { orthodontic* OR "orthodontic treatment") } \\
\text { \#2 TS }=(\text { "White lesions"OR "White spot lesions"OR "White spots"OR demineraliz* OR decalcif* OR remineraliz*) } \\
\text { \#3 TS = (manag* OR reduc* OR prevent* OR revers*) } \\
\text { \#4 TS = ("systematic review" OR "meta-analysis") } \\
\text { \#5 \#1 AND \#2 AND \#3 AND \#4 }\end{array}$ & $\# 5=24$ \\
\hline $\begin{array}{l}\text { CINAHL via EBSCO } \\
\text { from inception up to November 30, } 2019 \\
\text { TX - All Text } \\
\text { with no limits }\end{array}$ & $\begin{array}{l}\text { (orthodontic* OR "orthodontic treatment") AND ("White lesions" OR "White spot lesions"OR "White spots" } \\
\text { OR demineraliz* OR decalcif* OR remineraliz*) AND (manag* OR reduc* OR prevent* OR revers*) AND } \\
\text { ("systematic review" OR "meta-analysis") }\end{array}$ & 15 \\
\hline $\begin{array}{l}\text { PubMed } \\
\text { from inception up to November 30, } 2019 \\
\text { All fields } \\
\text { with no limits }\end{array}$ & $\begin{array}{l}\text { (orthodontic* OR "orthodontic treatment") } \\
\text { AND ("White lesions"OR"White spot lesions"OR"White spots"OR demineraliz* OR decalcif* OR remineraliz*) } \\
\text { AND (manag* OR reduc* OR prevent* OR revers*) } \\
\text { AND ("systematic review" OR "meta-analysis") }\end{array}$ & 36 \\
\hline
\end{tabular}


The overall confidence of the results of a systematic review was reported according to the following categories:

- high confidence of the results - no or only one noncritical weakness was found;

- moderate confidence - more than one non-critical weakness was found;

- low confidence - one critical flaw with/without a noncritical weakness was found;

- critically low confidence - more than one critical flaw with/without a non-critical weakness was found.

The overall confidence of the results of the selected systematic reviews was rated electronically by filling out the online AMSTAR-2 checklist (https://amstar.ca/Amstar_Checklist.php).

\section{Choice of the best body of evidence}

The Jadad decision algorithm is a simple guide that was designed to interpret discordant reviews and to help decision-makers select the most relevant and valid ones from among them. ${ }^{12}$ In this review, when an intervention was addressed by numerous systematic reviews with conflicting results, the Jadad decision algorithm was applied independently by the 2 reviewers. The results were then compared in order to robustly determine the systematic review which provided the best available evidence.

\section{Results}

\section{Search results}

A total of 141 references were retrieved through the search. After removing duplicates, 82 references were screened for eligibility by titles and abstracts. As a result, 33 articles were considered potentially relevant, and therefore checked in depth. Ultimately, 13 reviews were included. The detailed literature search process is provided in Fig. 3. The excluded reviews are presented in Supplementary Table 2 with the reasons for exclusion.

\section{Characteristics of the selected reviews}

The 13 reviews included in this review were published between 2004 and 2019; 4 of them were integrated into meta-analyses.,13-15 The addressed adjunctive interventions were topical fluoride application (assessed by 10 systematic reviews), ${ }^{4,6,8,13-19}$ the application of casein phosphopeptide-amorphous calcium phosphate (CPP-ACP) or other casein derivatives (assessed by 4 systematic reviews), ${ }^{6,17,18,20}$ fluoride-releasing bonding materials (assessed by 4 systematic reviews), ${ }^{13-15,21}$ laser therapy (assessed by 1 systematic review), ${ }^{3}$ resin infiltration (assessed by 1 systematic review), ${ }^{6}$ and micro-abrasion (assessed by 1 systematic review). ${ }^{6}$ The characteristics of the systematic reviews are summarized in Table 1.

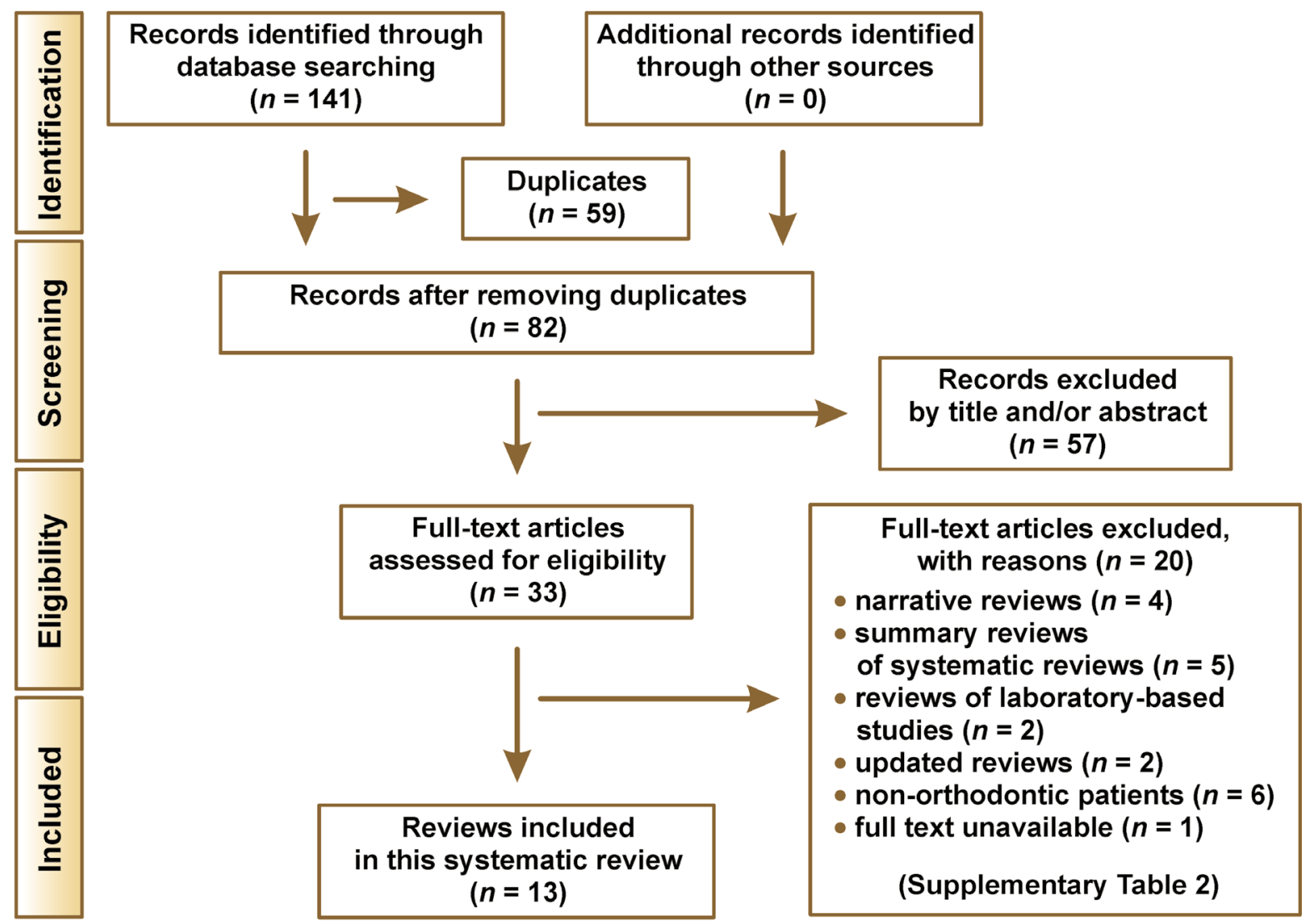

Fig. 3. Preferred Reporting Items for Systematic Reviews and Meta-Analyses (PRISMA) flow chart 
Supplementary Table 2. Excluded studies and the reasons for exclusion

\begin{tabular}{l|l|r} 
No. & Study & Reason for exclusion
\end{tabular}

Aravind N, Pandiyan S. Demineralisation around orthodontic brackets - A review. Res J Pharm Technol. 2016:9(9):1536-1540.

2 Raj BJR, Pradeep S. Remineralising agents in dentistry. Res J Pharm Technol. 2016;9(10):1734-1736.
Faghihian R, Shirani M, Tarrahi MJ, Zakizade M. Efficacy of the resin

3 infiltration technique in preventing initial caries progression: A systematic review and meta-analysis. Pediatr Dent. 2019;41(2):88-94.

Hani TB, O'Connell AC, Duane B. Casein phosphopeptide-amorphous

4 calcium phosphate products in caries prevention. Evid Based Dent. 2016;17(2):46-47.

Martinez-Mier EA. Fluoride-containing orthodontic adhesives may

5 reduce the occurrence of enamel demineralization in patients with fixed orthodontic appliances. J Evid Based Dent Pract. 2011;11(3):132-134.

6 Kalha AS. Topical fluorides and decalcification around fixed orthodontic appliances. Evid Based Dent. 2006;7(2):38-39. narrative review

narrative review

non-orthodontic patients

summary review of a systematic review

(Raphael S, Blinkhorn A. Is there a place for Tooth Mousse in the prevention and treatment of early dental caries? A systematic review. BMC Oral Health 2015;15(1):113.)

summary review of a systematic review

(Rogers S, Chadwick B, Treasure E. Fluoride-containing orthodontic adhesives and decalcification in patients with fixed appliances: A systematic review. Am J Orthod Dentofacial Orthop. 2010;138(4):390.e1-e390.e8.

summary review of a systematic review

(Chadwick BL, Roy J, Knox J, Treasure ET. The effect of topical fluorides on decalcification in patients with fixed orthodontic appliances: A systematic review. Am J Orthod Dentofacial Orthop. 2005;128(5):601-606.)

summary review of a systematic review

7 O'Neill J. Little evidence exists about optimal caries-prevention strategies during orthodontic treatment. Evid Based Dent. 2004;5(4):97.

Cheng LL. Limited evidence indicates fluoride may prevent

8 demineralized white lesions during orthodontic treatment. J Am Dent Assoc. 2015;146(9):699-701.

9 Taha AA, Patel MP, Hill RG, Fleming PS. The effect of bioactive glasses on enamel remineralization: A systematic review. J Dent. 2017;67:9-17.

Rahimi F, Sadeghi M, Mozaffari HR. Efficacy of fluoride varnish for prevention

10 of white spot lesions during orthodontic treatment with fixed appliances: A systematic review study. Biomed Res Ther. 2017:4(8):1513-1526.

Cosma LL, Şuhani RD, Mesaroş A, Badea ME. Current treatment

11 modalities of orthodontically induced white spot lesions and their outcome - a literature review. Med Pharm Rep. 2019;92(1):25-30.

Bergstrand F, Twetman S. Evidence for the efficacy of various methods

12 of treating white-spot lesions after debonding of fixed orthodontic appliances. J Clin Orthod. 2003;37(1):19-21.

Li J, Xie X, Wang Y, et al. Long-term remineralizing effect of casein

13 phosphopeptide-amorphous calcium phosphate (CPP-ACP) on early caries lesions in vivo: A systematic review. J Dent. 2014:42(7):769-777.

14 Paula ABP, Fernandes AR, Coelho AS, et al. Therapies for white spot lesions - a systematic review. J Evid Based Dent Pract. 2017;17(1):23-38.

Raphael S, Blinkhorn A. Is there a place for Tooth Mousse in the

15 prevention and treatment of early dental caries? A systematic review. BMC Oral Health. 2015:15(1):113.

Borges AB, Caneppele TMF, Masterson D, Maia LC. Is resin infiltration an

16 effective esthetic treatment for enamel development defects and white spot lesions? A systematic review. J Dent. 2017;56:11-18.

Indrapriyadharshini K, Madan Kumar PD, Sharma K, lyer K. Remineralizing

17 potential of CPP-ACP in white spot lesions - a systematic review. Indian J Dent Res. 2018;29(4):487-496.

Benson PE, Parkin N, Millett DT, Dyer F, Vine S, Shah A. Fluorides for

18 the prevention of white spots on teeth during fixed brace treatment. Cochrane Database Syst Rev. 2004;3:CD003809.

Sudjalim TR, Woods MG, Manton DJ. Prevention of white spot

19 lesions in orthodontic practice: A contemporary review. Aust Dent J. 2006;51(4):284-289;quiz 347

Bergstrand F, Twetman S. A review on prevention and treatment of

20 post-orthodontic white spot lesions - evidence-based methods and emerging technologies. Open Dent J. 2011;5:158-162. narrative review

neither abstract nor full-text available

(a request was sent to the corresponding author and the journal to get the article; however, no response was received)

non-orthodontic patients

non-orthodontic patients

non-orthodontic patients

non-orthodontic patients

non-orthodontic patients

updated in 2013; the updated version is included in this review

narrative review

updated in 2016; the updated version is included in this review 
Table 1. Characteristics of the included reviews assessing the effectiveness of different interventions in managing orthodontically induced white spot lesions (OIWSLs)

\begin{tabular}{|c|c|c|c|c|c|}
\hline Study/setting & Study design & $\begin{array}{l}\text { No. of primary } \\
\text { studies }\end{array}$ & Age of participants* & $\begin{array}{l}\text { Interventions/ } \\
\text { No. of studies in each }\end{array}$ & No. of participants \\
\hline $\begin{array}{l}\text { Tasios et al. }{ }^{15} \\
2019, \text { Greece }^{2}\end{array}$ & $\begin{array}{l}\text { systematic } \\
\text { review and } \\
\text { meta-analysis }\end{array}$ & 23 & $\begin{array}{l}\text { mean age of } \\
14.4 \text { years }\end{array}$ & $\begin{array}{l}\text { - fluoride varnish (4) } \\
\text { - fluoride varnish under banding } \\
\text { cement (2) } \\
\text { - fluoride-releasing adhesives ( } 8 \text { ) } \\
\text { - flat-surface sealants (5) } \\
\text { - active reminders (4) }\end{array}$ & 1,473 in total \\
\hline $\begin{array}{l}\text { Sardana et al. }{ }^{16} \\
\text { 2019, China }\end{array}$ & $\begin{array}{l}\text { systematic } \\
\text { review }\end{array}$ & 3 & $\begin{array}{c}\text { mean age of } \\
13.3 \text { years (for } 1 \text { study) } \\
\text { range of } \\
11-16 \text { years } / 13-21 \text { years } \\
\text { (for } 2 \text { studies) }\end{array}$ & self-applied topical fluorides & $\begin{array}{c}505 \text { (prevention of OIWSLs) } \\
26 \text { (reversal of OIWSLs ) }\end{array}$ \\
\hline $\begin{array}{l}\text { Pithon et al. }{ }^{20} \\
\text { 2019, Brazil }\end{array}$ & $\begin{array}{l}\text { systematic } \\
\text { review }\end{array}$ & 11 & $\begin{array}{l}\text { mean age of } \\
14.87 \text { years }\end{array}$ & CPP-ACP & not addressed \\
\hline $\begin{array}{l}\text { Sardana et al. }{ }^{4} \\
\text { 2019, China }\end{array}$ & $\begin{array}{l}\text { systematic } \\
\text { review and } \\
\text { meta-analysis }\end{array}$ & 11 & $\begin{array}{l}\text { mean age of } \\
15.21 \text { years }\end{array}$ & $\begin{array}{l}\text { professional topical fluoride } \\
\text { application in order to prevent } \\
\text { or reverse OIWSLs }\end{array}$ & $\begin{array}{l}518 \text { (prevention of OIWSLs) } \\
693 \text { (reversal of OIWSLs) }\end{array}$ \\
\hline $\begin{array}{l}\text { Raghis et al. }{ }^{3} \\
\text { 2018, Syria }\end{array}$ & $\begin{array}{l}\text { systematic } \\
\text { review }\end{array}$ & 8 & $\begin{array}{l}\text { mean age of } \\
13.45 \text { years }\end{array}$ & $\begin{array}{l}\text { laser irradiation: } \\
\text { - } \mathrm{CO}_{2} \text { laser } \\
\text { - } \mathrm{Nd} \text { :YAG laser } \\
\text { - argon laser } \\
\text { - Optodan laser }\end{array}$ & 183 \\
\hline $\begin{array}{l}\text { Nascimento et al. }{ }^{13} \\
\text { 2016, Brazil }\end{array}$ & $\begin{array}{l}\text { systematic } \\
\text { review and } \\
\text { meta-analysis }\end{array}$ & 7 & NA & $\begin{array}{l}\text { fluoride-releasing materials } \\
\text { (cementation materials, } \\
\text { varnish and sealant) }\end{array}$ & 1,867 teeth \\
\hline $\begin{array}{l}\text { Lapenaite et al. }{ }^{17} \\
\text { 2016, Lithuania }\end{array}$ & $\begin{array}{l}\text { systematic } \\
\text { review }\end{array}$ & 11 & NA & $\begin{array}{l}\text { fluoride products or casein } \\
\text { derivatives }\end{array}$ & $\begin{array}{c}998 \text { (studies on fluoride) } \\
314 \text { (studies on casein derivatives) }\end{array}$ \\
\hline $\begin{array}{l}\text { Sonesson et al. }{ }^{6} \\
\text { 2016, Denmark }\end{array}$ & $\begin{array}{l}\text { systematic } \\
\text { review }\end{array}$ & 7 & $\leq 30$ years & $\begin{array}{l}\text { - remineralizing agents } \\
\text { (fluoride, CCP-ACP) } \\
\text { - micro-abrasion } \\
\text { - resin infiltration }\end{array}$ & not addressed \\
\hline $\begin{array}{l}\text { Chen et al. }{ }^{18} \\
\text { 2013, China }\end{array}$ & $\begin{array}{l}\text { systematic } \\
\text { review }\end{array}$ & $\begin{array}{c}7 \\
\text { (3 - fluoride vs control; } \\
\text { 2- CPP-ACP vs control; } \\
\text { 2- fluoride vs CPP-ACP) }\end{array}$ & NA & $\begin{array}{l}\text { remineralizing agents } \\
\text { (fluoride, CCP-ACP) }\end{array}$ & $\begin{array}{c}173 \text { (studies on fluoride) } \\
110 \text { (studies on CPP-ACP) } \\
86 \text { (studies on fluoride vs CPP-ACP) }\end{array}$ \\
\hline $\begin{array}{l}\text { Benson et al. }{ }^{8} \\
\text { 2013, UK }\end{array}$ & $\begin{array}{l}\text { systematic } \\
\text { review }\end{array}$ & 3 & $\begin{array}{l}\text { mean age of } 15 \text { years } \\
\text { (for } 2 \text { studies) } \\
\text { adults up to } 45 \text { years } \\
\quad \text { (for } 1 \text { study) }\end{array}$ & fluorides & 458 \\
\hline $\begin{array}{l}\text { Rogers et al. }{ }^{21} \\
\text { 2010, UK }\end{array}$ & $\begin{array}{l}\text { systematic } \\
\text { review }\end{array}$ & 10 & NA & fluoride-containing adhesives & not addressed \\
\hline $\begin{array}{l}\text { Chadwick et al. }{ }^{19} \\
\text { 2005, UK }\end{array}$ & $\begin{array}{l}\text { systematic } \\
\text { review }\end{array}$ & 6 & NA & topical fluorides & not addressed \\
\hline $\begin{array}{l}\text { Derks et al. }{ }^{14} \\
\text { 2004, the Netherlands }\end{array}$ & $\begin{array}{l}\text { systematic } \\
\text { review and } \\
\text { meta-analysis }\end{array}$ & 15 & NA & $\begin{array}{l}\text { fluorides, chlorhexidine, } \\
\text { polymeric coating } \\
\text { or fluoride-releasing sealant, } \\
\text { and bonding material }\end{array}$ & $\begin{array}{c}847 \text { (fluorides) } \\
111 \text { (chlorhexidine) } \\
59 \text { (polymeric coating } \\
\text { or fluoride-releasing sealant) } \\
297 \text { (bonding material) }\end{array}$ \\
\hline
\end{tabular}

CPP-ACP - casein phosphopeptide-amorphous calcium phosphate; Nd:YAG laser - neodymium-doped yttrium aluminum garnet laser;

QLF - quantitative light-induced fluorescence; $M$ - mean; SD - standard deviation; $\mathrm{Cl}$ - confidence interval;

SEM - scanning electron microscopy; NA - not applicable.

* The mean age of the participants was calculated based on the available data (mean age) provided in the included reviews. 


\begin{tabular}{|c|c|c|}
\hline Outcomes & \begin{tabular}{c|} 
Quality of \\
primary studies
\end{tabular} & Results \\
\hline $\begin{array}{l}\text { - incidence of OIWSLS } \\
\text { - severity of OIWSLS }\end{array}$ & $\begin{array}{l}\text { unclear risk (7) } \\
\text { high risk (16) }\end{array}$ & $\begin{array}{l}\text { - Fluoride varnish, flat-surface sealants and active reminders were associated with a reduced development } \\
\text { and magnitude of lesions; however, the strength of this recommendation is mostly low. } \\
\text { - No statistically significant difference was found for fluoride varnish under banding cement. } \\
\text { - No statistically significant improvement was found for fluoride-releasing adhesives. }\end{array}$ \\
\hline $\begin{array}{l}\text { - changes in the incidence of OIWSLs as the } \\
\text { primary outcome (studies on prevention) } \\
\text { - reduction in the size of OIWSLs or mean } \\
\text { fluorescence values or the prevalence of OIWSLS } \\
\text { as the primary outcomes (studies on reversal) }\end{array}$ & $\begin{array}{l}\text { low risk(1) } \\
\text { high risk (2) }\end{array}$ & $\begin{array}{l}\text { Although the review did not confirm the effectiveness of self-applied fluorides in the reversal of OIWSLS, } \\
\text { it did partially substantiate the positive role (although indirectly) of self-applied fluorides } \\
\text { in the prevention of OIWSLs based on a single trial with a low risk of bias. }\end{array}$ \\
\hline $\begin{array}{c}\text { prevention or treatment of OIWSLs around } \\
\text { orthodontic braces }\end{array}$ & $\begin{array}{l}\text { low risk (9) } \\
\text { unclear risk (2) }\end{array}$ & The CPP-ACP products were effective in the prevention and treating of OIWSLs. \\
\hline $\begin{array}{l}\text { - difference in the incidence of OIWSLS } \\
\text { - change in the mean QLF scores or the mean } \\
\text { DIAGOdent scores }\end{array}$ & $\begin{array}{l}\text { low risk (5) } \\
\text { medium risk (4) } \\
\text { high risk (2) }\end{array}$ & $\begin{array}{l}\text { - Professional topical fluoride application brought about a } 25-30 \% \text { reduction in the incidence of OIWSLs } \\
\text { (pooling } 3 \text { studies). } \\
\text { - The standardized mean difference was found to be } 0.57 \text { less in the professional topical fluoride application } \\
\text { group than in the control group ( } 95 \% \text { Cl: from } 0.23 \text { to - } 0.91 \text { ) in the studies evaluating the reversal of } \\
\text { OIWSLs (pooling } 3 \text { studies); however, the effect of professional topical fluoride application on the reversal } \\
\text { of OIWSLs was unclear due to concerns in interpreting the DIAGNOdent values. }\end{array}$ \\
\hline $\begin{array}{l}\text { - formation or non-formation of OIWSLs assessed } \\
\text { clinically or with digital images } \\
\text { - degree of decalcification } \\
\text { - changes in the enamel structure after laser } \\
\text { application }\end{array}$ & $\begin{array}{l}\text { unclear risk } \\
\text { (all studies) }\end{array}$ & Laser irradiation was effective in inhibiting demineralization during orthodontic treatment. \\
\hline $\begin{array}{l}\text { - risk of patients developing OIWSLs } \\
\text { - the } M \text { and } S D \text { values of the extent of OIWSLs } \\
\text { acc. to the Gorelick scale }\end{array}$ & $\begin{array}{l}\text { high risk } \\
\text { (all studies) }\end{array}$ & $\begin{array}{l}\text { - The pooled relative risk of developing OIWSLs when using cementation materials as compared to } \\
\text { control was } 0.35 \text { ( } 95 \% \text { CI: } 0.15-0.81 \text { ); hence, patients using fluoride-releasing materials were at a lower } \\
\text { risk of developing OIWSLs (pooling } 4 \text { studies). } \\
\text { The risk reduction was statistically significant for fluoride varnish, but not for sealant (only } 1 \text { study was } \\
\text { included for each intervention). } \\
\text { - The pooled mean difference in the extent of OIWSLs between the experimental and control groups was } \\
\text { statistically significant for cementation materials, but not for varnish and sealant. }\end{array}$ \\
\hline prevention of OIWSLS & not addressed & $\begin{array}{l}\text { The results showed positive effects of the fluoride products and casein derivatives on preventing OIWSLS } \\
\text { ( } 4 \text { out of } 6 \text { studies revealed the effectiveness of the fluoride products in preventing OIWSLs; } \\
3 \text { out of } 5 \text { studies revealed the effectiveness of casein derivatives in preventing OIWSLs). }\end{array}$ \\
\hline $\begin{array}{l}\text { difference in the severity of OIWSLs between the } \\
\text { experimental and control groups as the primary } \\
\text { outcome (severity was expressed in terms of the } \\
\text { area over the whiteness of the lesion or as the } \\
\text { amount of mineral loss or the lesion depth) }\end{array}$ & $\begin{array}{l}\text { medium risk (1) } \\
\text { high risk (6) }\end{array}$ & $\begin{array}{l}\text { There is a lack of reliable evidence to support the effectiveness of remineralizing agents in the treatment } \\
\text { of OIWSLS (1 out of } 3 \text { studies revealed the ineffectiveness of fluorides in managing OIWSLs; } 1 \text { study } \\
\text { indicated that CPP-ACP was effective in managing OIWSLs, while another one found no significant } \\
\text { difference as compared to control; } 1 \text { study indicated that fluoride was more effective than CPP-ACP, while } \\
\text { another one found no difference). }\end{array}$ \\
\hline $\begin{array}{l}\text { - presence or absence of new lesions by participant } \\
\text { - differences in the size and severity of OIWSLS } \\
\text { between the experimental and control groups } \\
\text { - any quantitative assessment of enamel mineral } \\
\text { loss, such as fluorescent light techniques or } \\
\text { microradiography, used with in situ caries } \\
\text { models } \\
\text { - any participant-assessed outcomes, such as the } \\
\text { perception of the lesions and oral health-related } \\
\text { quality of life data } \\
\text { - adverse effects }\end{array}$ & $\begin{array}{l}\text { low risk (1) } \\
\text { medium risk (1) } \\
\text { high risk (1) }\end{array}$ & $\begin{array}{l}\text { - Applying fluoride varnish every } 6 \text { weeks at the time of the orthodontic review during treatment is effective, } \\
\text { but this finding is based on a single study. } \\
\text { - None of the included studies reported data on the size and severity of OIWSLs, the quantitative assessment } \\
\text { of enamel mineral loss, patient's perception, oral health-related quality of life, and adverse effects. }\end{array}$ \\
\hline $\begin{array}{l}\text { any outcome measures of decalcification } \\
\text { and the presence of OIWSLs }\end{array}$ & not addressed & $\begin{array}{l}\text { It is impossible to make recommendations on the use of fluoride-containing adhesives during fixed } \\
\text { orthodontic treatment. However, there is evidence to suggest that glass ionomer cement is more effective } \\
\text { than composite resin in the prevention of OIWSLs, but the evidence is weak. }\end{array}$ \\
\hline $\begin{array}{l}\text { any valid outcome measure of decalcification, } \\
\text { both before and after treatment }\end{array}$ & not addressed & $\begin{array}{l}\text { The use of topical fluorides in addition to a fluoride toothpaste reduced the incidence of decalcification } \\
\text { in populations with both fluoridated and non-fluoridated water supplies (all the included studies found } \\
\text { a significant difference). Different preparations and formats appear to decrease decalcification, but there is } \\
\text { no evidence that any method is superior. There is some evidence that the potency of fluoride preparations } \\
\text { might be important. }\end{array}$ \\
\hline any demineralization scores & not addressed & $\begin{array}{l}\text { The use of a toothpaste and gel with a high fluoride concentration of 1,500-5,000 ppm, or } \\
\text { complementary chlorhexidine during orthodontic treatment showed a demineralization-inhibiting effect. } \\
\text { The use of a polymeric tooth coating on the tooth surface around the brackets or a fluoride-releasing } \\
\text { bonding material showed almost no demineralization-inhibiting effect. Pooling data was only applied for } \\
\text { the studies assessing fluoride-releasing bonding materials, where the overall prevented fraction was } 20 \% \\
\text { (SEM 0.09). However, this effect was not statistically significant. }\end{array}$ \\
\hline
\end{tabular}




\section{Methodological quality of the included reviews}

The methodological quality of the reviews ranged between a critically low to moderate confidence of the results. In other words, all the reviews were fraught with some methodological shortcomings. The AMSTAR-2 questions and the results for the reviews are presented in Table 2. The detailed supporting reasons for each assessment are provided in Supplementary Table 3. With regard to the 7 critical domains of the AMSTAR-2 tool, most reviews failed to contain an explicit statement that the review methods were established prior to the conduct of the review (69.23\% of the included reviews), did not use a satisfactory technique to assess the risk of bias in each individual study (53.84\% of the included reviews), and did not account for the risk of bias in individual studies when interpreting and discussing the results $(46.15 \%$ of the included reviews).

\section{Effects of interventions}

\section{Effect of topical fluorides in the management of OIWSLs}

Ten systematic reviews provided the results from studies evaluating the effectiveness of topical fluorides in the management of OIWSLs: Tasios et al. 2019, ${ }^{15}$ Sardana et al. 2019, ${ }^{16}$
Sardana et al. 2019 (Epub 2018), ${ }^{4}$ Nascimento et al. 2016, ${ }^{13}$ Lapenaite et al. 2016, ${ }^{17}$ Sonesson et al. 2016, ${ }^{6}$ Chen et al. 2013, ${ }^{18}$ Benson et al. 2013, ${ }^{8}$ Chadwick et al. 2005, ${ }^{19}$ and Derks et al. 2004. ${ }^{14}$ Different forms were utilized to apply topical fluorides, including fluoride varnish, fluoride gel, fluoridated mouth rinse, fluoridated toothpaste, fluoridated water, and fluoride chewing sticks.

Some of these reviews deduced that topical fluorides were effective in managing OIWSLs, ${ }^{4,8,13-15,17,19}$ while others failed to find reliable significant evidence. ${ }^{6,16,18}$ All the reviews were addressing the same review question, but the recruited trials and the selection criteria varied among them. In this case, the Jadad algorithm suggests that the best available review be selected according to the superiority in its publication status, the methodological quality of its primary studies, language restrictions, and the analysis of data on individual patients. As a result, Sardana et al. $2019^{4}$ was selected (Fig. 4). The detailed steps of the Jadad decision algorithm used from the beginning until the final constructive decision are presented in Supplementary Table 4.

Sardana et al. $2019^{4}$ addressed the use of professional fluorides and recruited 11 RCTs published between 2007 and 2017. All primary studies used a parallel-arm design. The results indicated that fluorides led to a $25-30 \%$ reduction in the incidence of OIWSLs; however, their effect on reversing lesions was unclear due to concerns in interpreting the DIAGNOdent values.

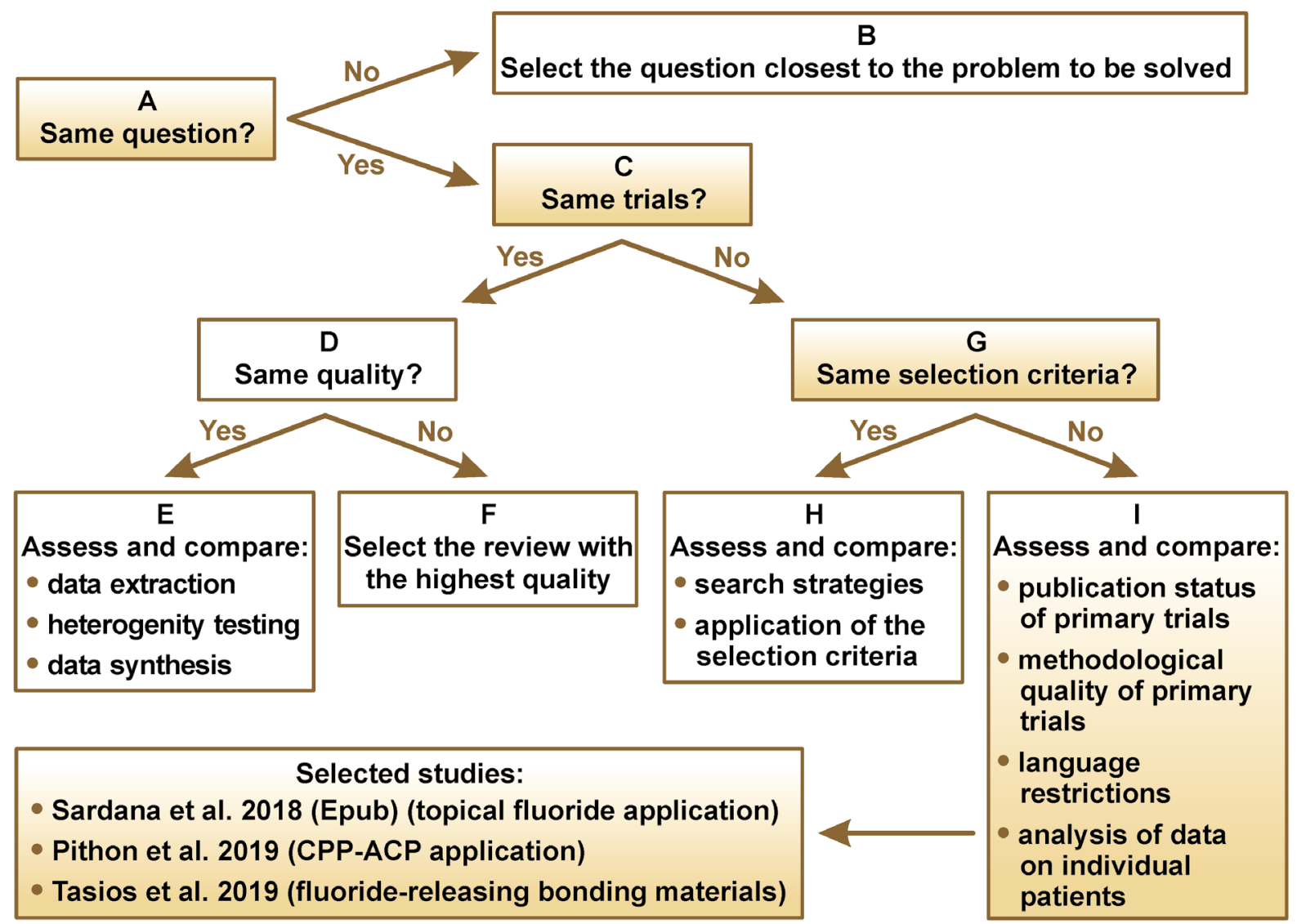

Fig. 4. Flow diagram of the Jadad decision algorithm for conflicting reviews on efficacy of topical fluorides, casein phosphopeptide-amorphous calcium phosphate (CPP-ACP) and fluoride-releasing adhesives 


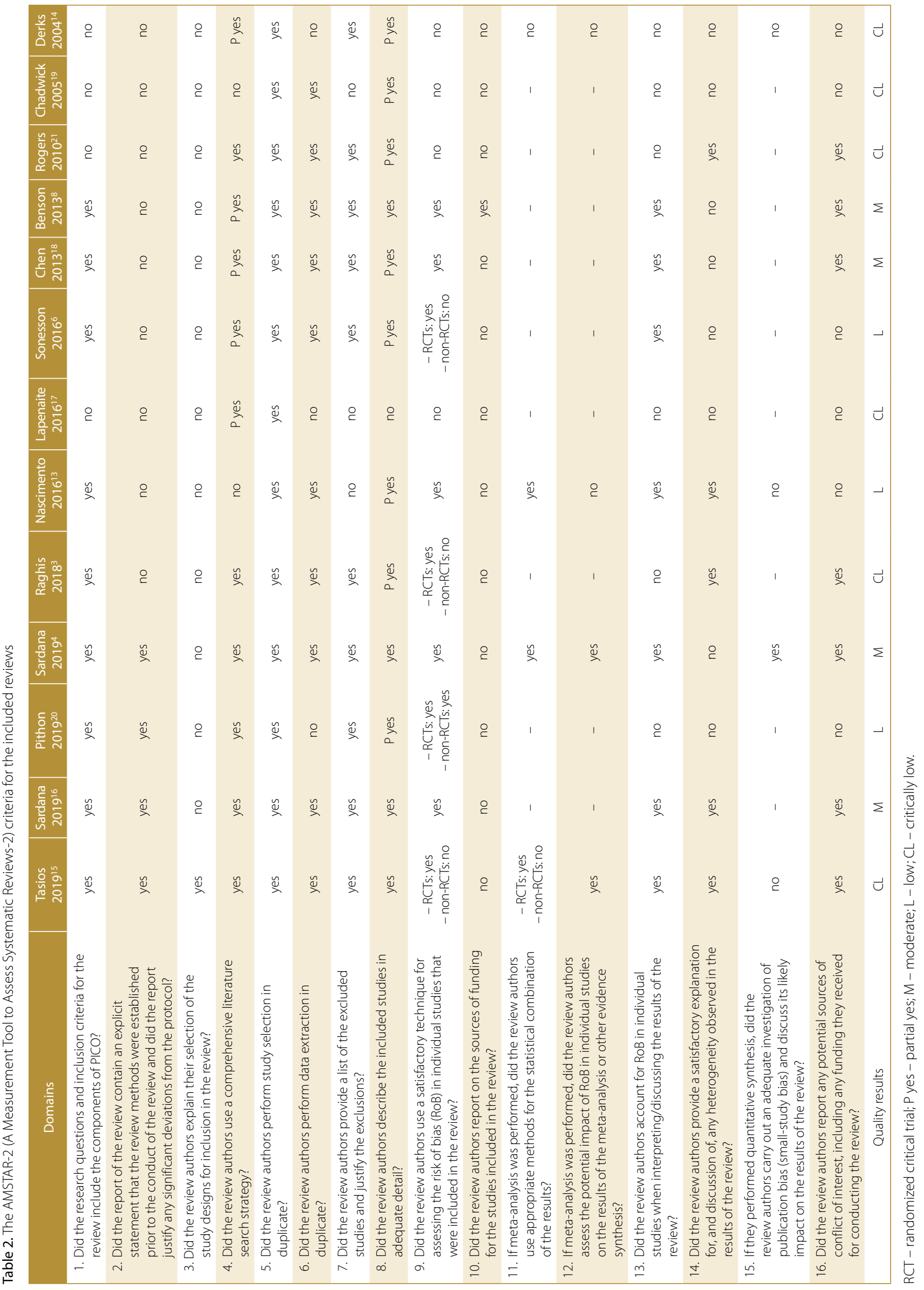


Supplementary Table 3. Detailed supporting reasons for the AMSTAR-2 (A Measurement Tool to Assess Systematic Reviews-2) assessment of each included review

\begin{tabular}{|c|c|}
\hline Domains & Tasios $2019^{15}$ \\
\hline $\begin{array}{l}\text { 1. Did the research questions and } \\
\text { inclusion criteria for the review include the } \\
\text { components of PICO? }\end{array}$ & $\begin{array}{l}\text { Yes: As stated on page } 2 \text { in the "Eligibility criteria"section: "According } \\
\text { to the Participants-Intervention-Comparison-Outcome study design } \\
\text { (PICOS)..." }\end{array}$ \\
\hline $\begin{array}{l}\text { 2. Did the report of the review contain an } \\
\text { explicit statement that the review methods } \\
\text { were established prior to the conduct of } \\
\text { the review and did the report justify any } \\
\text { significant deviations from the protocol? }\end{array}$ & $\begin{array}{l}\text { Yes: As stated on page 2: "The review protocol was made a priori, } \\
\text { registered in PROSPERO (CRD42017079352) and all post hoc changes } \\
\text { were appropriately noted". }\end{array}$ \\
\hline $\begin{array}{l}\text { 3. Did the review authors explain their } \\
\text { selection of the study designs for inclusion } \\
\text { in the review? }\end{array}$ & $\begin{array}{l}\text { Yes: As stated on page 2: "due to the scarcity of RCTs on this subject, } \\
\text { included were randomized or quasi-randomized prospective } \\
\text { controlled human trials". }\end{array}$ \\
\hline $\begin{array}{l}\text { 4. Did the review authors use a } \\
\text { comprehensive literature search strategy? }\end{array}$ & $\begin{array}{l}\text { Yes: } \\
\text { - Authors searched } 9 \text { databases. } \\
\text { - Authors searched trial registries: metaRegister of Controlled Trials. } \\
\text { - Authors searched DOAJ and PQDT. } \\
\text { - The reference lists and Google Scholar citation lists of the eligible } \\
\text { full-text articles as well as the reference lists of relevant systematic } \\
\text { reviews were screened manually for additional studies. } \\
\text { - Key words and the search strategy were provided in Appendix Table } 1 \text {. } \\
\text { - No restrictions were applied on the language, publication year or } \\
\text { status. }\end{array}$ \\
\hline
\end{tabular}

5. Did the review authors perform study selection in duplicate?

6. Did the review authors perform data extraction in duplicate?

7. Did the review authors provide a list of the excluded studies and justify the exclusions?

8. Did the review authors describe the included studies in adequate detail?

9. Did the review authors use a satisfactory technique for assessing the risk of bias (RoB) in individual studies that were included in in individual stu

10. Did the review authors report on the sources of funding for the studies included in the review?

11. If meta-analysis was performed, did the review authors use appropriate methods for the statistical combination of the results?

12. If meta-analysis was performed, did the review authors assess the potential impact of RoB in individual studies on the results of the meta-analysis or other evidence synthesis?

13. Did the review authors account for RoB in individual studies when interpreting/ discussing the results of the review?

Yes: The risk of bias in individual studies was accounted for, especially when applying the GRADE criteria.

14. Did the review authors provide a satisfactory explanation for, and

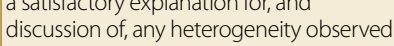
in the results of the review?

Yes: As stated in the abstract: "Study selection, data extraction and risk of bias assessment were done independently in duplicate".

Yes: As stated in the abstract: "Study selection, data extraction and risk of bias assessment were done independently in duplicate".

Yes: The list of the studies identified from the literature and their inclusion/exclusion status with reasons was provided in Appendix Table 2. design, the study setting, and follow-up were all described in details in Tables 1 and 2 as well as in the text.

Yes: Randomized controlled trials were appropriately assessed by the RoB tool.

No: Non-randomized controlled trials were assessed by the RoB tool rather than the ROBINS-I tool.

No: Funding for primary studies was not reported in the review or in the "Other bias" section referring to the RoB tool.

Yes: For RCTs, authors used an appropriate technique to combine the study results and adjusted for heterogeneity.

No: Authors did not report separate summary estimates for NRSIs.

Yes: Authors performed sensitivity analyses and presented them in Appendix Table 8. the results of the review.
Yes: Populations, interventions, comparators, outcomes, the study

Meta-analysis was not performed

Yes: The risk of bias was accounted for when interpreting results such as: "Since this deduction is made based on 2 trials -1 with a low risk of overall bias and 1 with a high risk of bias - this conclusion should be interpreted with caution, and the present review recommends further trials to confirm this". Moreover, the risk of bias was accounted for when evaluating the overall quality of evidence.

Yes: As stated: "All 3 trials were quite heterogeneous in regards to the intervention (high-fluoride vs low-fluoride toothpaste; the composition of the fluoride agent), control group and outcome composition of the fluoride agent), control group and outcome
( 2 assessed prevention at debonding and 1 assessed reversal at Yes: The impact of heterogeneity was accounted for when discussing 26 weeks). Hence, it is quite difficult to give any recommendation by pooling the results qualitatively" and "The trials included in the review were of considerable heterogeneity, and this hampers providing the recommendations/quidelines pertaining to the concentration of

fluoride, the fluoride carrier or the fluoride agent in the carrier".

15. If they performed quantitative synthesis, did the review authors carry out an adequate investigation of publication bias (small-study bias) and discuss its likely impact on the results of the review?

16. Did the review authors report any potential sources of conflict of interest, including any funding they received for conducting the review? 2 authors (DS and MS) independently screened titles and their inclusion/exclusion in the review".
incts in a standardized manner to dete

Yes: As stated on page 3: "The characteristics of the respective studies on a piloted pro forma"

Yes: As stated on page 3:"The detailed reasons for the exclusion of the

Yes: Populations, interventions, comparators, outcomes, the study design, the study setting, and follow-up were all described in Table 2 .

Yes: Randomized controlled trials were appropriately assessed by the

Non-randomized trials were not included.

No: Funding for primary studies was not reported in the review.

Meta-analysis was not performed

No: Publication bias was not considered in the review.

Meta-analysis was not performed

Yes: As stated on page 10: "All authors declare that there is no conflict of interest".
Yes: As stated in the abstract: "This research did not receive any specific grant from funding agencies in the public, commercial or not-for-profit sectors".
Yes: As stated on page 2:"The Population-Intervention-Controlas follows:..."

Yes: As stated on page 2: "The methodology of the review was formulated in advance by strictly adhering to the PRISMA guidelines".

No: Authors did not provide the explanation for including RCTs and quasi-randomized CCTs only.

Authors searched 4 databases.

registered trial or unpublished material.

The reference lists of the eligible articles were screened manually for

Key words were provided in Table 1.

No restrictions were applied on the publication year or status.

Yes: As stated on page 3:"After the removal of duplicates, 
Continued Supplementary Table 3

\section{Pithon $2019^{20}$}

Yes: As stated on page 2: "To be included in the review,

1. studies needed to comply with the following selection criteria, according to the PICOS strategy..."

Yes: As stated on page 2:"The protocol used for the systematic review of the effectiveness of CPP-ACP-

2. containing products was recorded in the International Prospective Register of Systematic Reviews".

3. No: Authors did not provide the explanation for including both RCTs and CCTs.

\section{Yes:}

- Authors searched 5 databases.

- Authors searched trial registries: ClinicalTrials.

- Grey literature was searched by www.opengrey.com.

4. - The reference lists of the eligible articles were screened manually for additional studies.

- Key words were provided within the text.

- No restrictions were applied on the language, publication year or status.

5. Yes: As stated on page 2: "The selections were performed by 2 independent researchers"

6. No: It was not stated that data was extracted in duplicate

Yes: The potentially relevant studies that were read in the full-text form, but excluded from the review were stated and cited within the text in the "Results" section (page 3) with the justification for exclusion.

Partial Yes: Populations, interventions, comparators,

8. outcomes, and the study design were described. However, the study setting and follow-up were not addressed.

Yes: Randomized controlled trials were appropriately assessed by the RoB-2 tool.

9. Yes: Non-randomized controlled trials were appropriately assessed by the ROBINS-I tool.

No: Funding for primary studies was not reported in the review.

No: Funding for primary studies was not reported in
the review.

Yes: Authors used an appropriate weighted

technique to combine the study results and adjusted for heterogeneity if present

Yes: Sensitivity analysis was performed by excluding 'high-risk' trials and trials with 'some concerns'from quantitative synthesis.

No: Authors did not account for the moderate risk of

13. bias found in the 2 included studies when interpreting the results.

14. No: Heterogeneity was not addressed

discussed its impact on the results of the review.

Yes: Though funnel plots and statistical analysis were not provided, publication bias was discussed in the review as follows: "the unpublished study did not find any significant effect of $1.23 \%$ APF gel on the prevention of EWSLs during orthodontic treatment, which might have resulted in publication bias".

Yes: As stated on page 9: "This research did not receive any specific grant from funding agencies in the public, commercial or not-for-profit sectors.

Conflicts of interest: None to be declared".
16. in the review.

\section{Raghis $2018^{3}$}

Yes: As stated on page 323: "These review questions were developed according to the population, intervention, comparison, and outcome (PICO) study design.

No: The review did not contain an explicit statement that the review methods were established prior to the conduct of the review.

No: Authors did not provide the explanation for including both RCTs and CCTs.

Yes:

- Authors searched 5 databases.

- Authors searched trial registries: ClinicalTrials and ICTRP.

- Grey literature was searched by www.opengrey.com.

- PQDT was searched for dissertations.

- The reference lists of the eligible articles were screened manually for additional studies.

- Key words and the search strategy were provided in Supplementary Material 1.

- No restrictions were applied on the language,

publication year or status.

Yes: As stated on page 323: "The obtained articles were independently subjected to clear inclusion and exclusion criteria by 2 authors."

Yes: As stated on page 323: "The data was extracted from studies according to the aims of the systematic review by the same 2 authors independently".

Yes: The details of the reasons for the exclusion of studies after full-text reading was presented in Supplementary Material 2

Partial Yes: Though populations, interventions, comparators, outcomes, the study design, and follow-up were described, the study setting was not addressed.

Yes: Randomized controlled trials were appropriately assessed by the RoB tool.

No: Non-randomized controlled trials were assessed by the RoB tool rather than the ROBINS-I tool.

No: Funding for primary studies was not reported in the review or in the "Other bias" section referring to the RoB tool.

Meta-analysis was not performed.

Meta-analysis was not performed.

No: Authors did not account for the unclear risk of bias found in all the included studies when interpreting the results.

Yes: As stated on page 325: "Heterogeneity was observed due to the study designs, treatment protocols and the assessed outcomes of the included studies".

Meta-analysis was not performed

Yes: As stated on page 321:"Funding: None declared. Conflict of interest: None declared". 


\section{Continued Supplementary Table 3}

\section{Nascimento $2016^{13}$}

Yes: As stated on page 102: "The eligibility criteria and

1. search strategy were based on the PICO elements (population, intervention, comparison, and outcome)".

No: The review did not contain an explicit statement

2. that the review methods were established prior to the conduct of the review.

No: Authors did not provide the explanation for including only RCTs.

\section{No:}

- Authors searched only 1 database.

- Trial registries and grey literature were not searched.

- The reference lists of the eligible articles were screened manually for additional studies.

- Key words were provided on page 102.

- No restrictions were applied on the language, publication year or status.

5. $\quad$ Yes: As stated on page 102: "Two independent reviewers screened the search results".

Yes: As stated on page 102: "To record the study characteristics, methodological quality and results, the reviewers used a data extraction form according to the CONSORT 2010 statement. Disagreement between the 2 reviewers was solved either by consensus or by a third reviewer".

No: The list of the excluded studies and the reasons

for exclusion were not provided. Figure 1 provided the

. number of the excluded studies without providing the authors' names for each study.

Partial Yes: Populations, interventions, comparators, outcomes, and the study design were described,

but not in details. Moreover, the study setting and follow-up were not addressed.

Yes: Randomized controlled trials were appropriately assessed by the RoB tool.

Not-randomized trials were not included. included when addressing the inclusion criteria (comparison and outcome were not stated). established prior to the conduct of the review.

No: Authors did not provide the explanation for

\section{Partial Yes:}

- Authors searched 5 databases.

- Trial registries and grey literature were not searched.

- The reference lists of the eligible articles were screened manually for additional studies.

- Key words were provided on page 4.

- Restrictions were applied on the language (only articles written in English) and publication year (articles published between 2008 and 2013)

Yes: As stated on page 4:"Three researchers independently reviewed the titles and abstracts of the potentially relevant studies".

\section{Lapenaite $2016^{17}$}

No: The PICO components were not all

No: The review did not contain an explicit statement that the review methods were including both RCTs and quasi-RCTs.

Sonesson $2016^{6}$

Yes: As stated on page 2: "The PICO was set up as follows..."

No: The review did not contain an explicit statement that the review methods were established prior to the conduct of the review.

No: Authors did not provide the explanation for including both RCTs and CCTs.

Partial Yes:

- Authors searched 4 databases.

- Authors searched trial registries: ClinicalTrials.

- Grey literature was not searched.

- The reference lists of the eligible articles were screened manually for additional studies.

- Key words and the search strategy were provided in Supplement 1.

- Restrictions were applied on the language (only articles written in English) and publication year (articles published between 2011 and Oct 31, 2015).

Yes: As stated in the abstract: "Abstract lists and selected full-text papers were independently examined by 2 reviewers, and any differences were solved by consensus".

No: The review did not contain an explicit statement that authors performed data extraction in duplicate.

Yes: As stated on page 2: "Key data from the accepted studies were extracted independently by 2 authors".
No: The list of the excluded studies and the reasons for exclusion were not provided.

No: Though populations, interventions and comparators were described (not in details) and follow-up were not addressed. outcomes, the study design, the study setting
Yes: As stated on page 3: "The excluded studies with the main reason for exclusion are shown in Table 3".
Partial Yes: Populations, interventions, comparators, outcomes, the study design, and follow-up were described, but not in details. Moreover, the study setting was not addressed.

Yes: Randomized controlled trials were appropriately

assessed by the RoB tool.
No: Non-randomized controlled trials were assessed

No: The risk of bias was not assessed by the RoB tool rather than the ROBINS-I too

10. No: Funding for primary studies was not reported in No: Funding for primary studies was not reported No: Funding for primary studies was not reported in 10. in the review. the review.

Yes: Authors used an appropriate technique

11. to combine the study results and adjusted for heterogeneity.

No: All studies were at a high risk of bias and were al included in evidence synthesis.

Meta-analysis was not performed.

Meta-analysis was not performed.

\section{No: All studies were at a high risk of bias and were all}

Yes: As stated:"all the included studies presented a

13. Yes: As stated: "all the included studies presented a
high risk of bias, indicating that the evidence is weak".

Yes: As stated on page 106: "The subgroup analysis showed a $65 \%$ heterogeneity between the materials, indicating that the type of material partially explained the heterogeneity. Thus, different approaches for delivering fluorides near brackets could be related to the observed heterogeneity".

15. No: Publication bias was not assessed

Meta-analysis was not performed.

No: The risk of bias was not assessed.
Meta-analysis was not performed.

Yes: The risk of bias in individual studies was accounted for when interpreting the results, especially when applying the GRADE criteria.

No: Authors just stated in the abstract that: "No pooling

No: Heterogeneity was not addressed.

of the results was possible due to study heterogeneity" without providing any further explanation.
16. No: Neither funding nor conflicting interests were stated in the review.
Meta-analysis was not performed.

Meta-analysis was not performed.

No: Though authors mentioned their funding sources No: Neither funding nor conflicting interests were "Funding: The project was funded through the authors" stated in the review. academic institutions", they did not explain how they managed the potential conflict of interest. 
Continued Supplementary Table 3

\section{Chen $2013^{18}$}

Yes: The PICO elements were mentioned on . page 377 in the "Material and methods" section.

No: The review did not contain an explicit

2. statement that the review methods were established prior to the conduct of the review.

3. No: Authors did not provide the explanation for including only RCTs.
Partial Yes:

- Authors searched 4 databases.

- Trial registries and grey literature were not searched.

4. The reference lists of the eligible articles were screened manually for additional studies.

- Key words and the search strategy were provided in Appendix 1

- No restrictions were applied on the language, publication year or status. relevant studies were scrutinized by 2 reviewers

5. (HC and TG) independently to select the eligible studies".

Yes: As stated on page 377: "Data from all the 6. eligible studies was extracted by 2 reviewers $(H C$ and TG) independently, in duplicate".

Yes: As stated on page 9: "Data was extracted by 2 review authors independently, in duplicate,

Yes: As stated on page 378: "The list of the 7. excluded articles and the reasons for exclusion are in Appendix II".

Partial Yes: Populations, interventions, 8. comparators, outcomes, the study design, and

follow-up were described, but not in details. Moreover, the study setting was not addressed.

Yes: Randomized controlled trials were appropriately assessed by the RoB tool. Not-randomized trials were not included.

10. No: Funding for primary studies was not reported in the review.

12. Meta-analysis was not performed.

Yes: The risk of bias of studies was discussed with

13. the reasons beyond this bias. Noteworthy, most trials were at a high risk of bias.

14. No: Heterogeneity was not addressed.
Yes: Authors provided the list of the excluded studies with the reasons for exclusion in Table "Characteristics of excluded studies".

Yes: Populations, interventions, comparators, outcomes, the study design, the study setting and follow up were all described in details in Table "Characteristics of included studies".

Yes: Randomized controlled trails were appropriately assessed by the RoB tool.

Non-randomized trials were not included.

Yes: The sources of funding for primary studies were reported in Table "Characteristics of included studies".
Meta-analysis was not performed.

Yes: The risk of bias in individual studies was accounted for several times when interpreting and discussing the results.

No: Though it was planned to measure heterogeneity, reviewers did not provide the
Yes: As stated on page 377: "The full texts of the using specially designed data extraction forms".

11. Meta-analysis was not performed.

Rogers $2010^{21}$

No: The PICO components were not all included when addressing the inclusion criteria.

No: The review did not contain an explicit statement that the review methods were established prior to the conduct of the review.

No: Authors did not provide the explanation for including all of the following: RCTs, clinical trials, and prospective observational studies with concurrent or historic comparison groups.

\section{Yes:}

- Authors searched 5 databases.

- Authors searched trial registries: Current Controlled Trials Register and National

Research Register.

- Grey literature was searched by SIGLE and ISI Conference Proceedings.

- The reference lists of the eligible articles were screened manually for additional studies.

- Key words were provided in Table 1.

- No restrictions were applied on the language, publication year or status.

Yes: As stated on page 390.e3: "all the selected articles were examined by 2 independent

reviewers ( $S R$ and $B C$ ) to determine whether the eligibility criteria were met".

Yes: As stated on page 390.e3: "data was extracted and methodological quality was assessed by 2 reviewers independently, in duplicate, using specially designed data extraction forms".

Yes: Authors mentioned within the text on page 390.e3 in the "Results" section the relevant studies that were excluded from the review with the justification for exclusion.

Partial Yes: Populations, interventions, comparators, outcomes, the study design, and follow-up were described, but not in details. Moreover, the study setting was not addressed.

No: The risk of bias was not assessed.

No: Funding for primary studies was not reported in the review.

Meta-analysis was not performed.

Meta-analysis was not performed. explanation for heterogeneity in the results, as meta-analysis was not conducted.

Meta-analysis was not performed

15. Meta-analysis was not performed. Yes: As stated on page 376: "The authors report no commercial, proprietary or financial interest in the products or companies described in this article
Yes: As stated on page 34: "No interests to declare".
No: The risk of bias was not assessed.

Yes: As stated on page 390.e5: "With regard to the materials used, the studies involved could be considered non-homogenous. The adhesives can all be described as containing fluoride, but they essentially have different chemical properties"

Meta-analysis was not performed.

Yes: As stated on page 390.e1: "The authors of this article report no commercial, proprietary or financial interest in the products or companies described in this article. 


\section{Continued Supplementary Table 3}

\begin{tabular}{|c|c|c|}
\hline & Chadwick $2005^{19}$ & Derks $2004^{14}$ \\
\hline 1. & $\begin{array}{l}\text { No: The PICO components were not all included when addressing the } \\
\text { inclusion criteria (comparison was not stated). }\end{array}$ & $\begin{array}{l}\text { No: The PICO components were not all included when addressing the } \\
\text { inclusion criteria. }\end{array}$ \\
\hline 2. & $\begin{array}{l}\text { No: The review did not contain an explicit statement that the review } \\
\text { methods were established prior to the conduct of the review. }\end{array}$ & $\begin{array}{l}\text { No: The review did not contain an explicit statement that the review } \\
\text { methods were established prior to the conduct of the review. }\end{array}$ \\
\hline 3. & $\begin{array}{l}\text { No: Authors did not provide the explanation for including all of the } \\
\text { following: RCTs, clinical trials, and prospective observational studies with } \\
\text { concurrent or historical comparison groups. }\end{array}$ & No: Authors did not provide the explanation for including only RCTs. \\
\hline 4. & $\begin{array}{l}\text { - No: } \\
\text { - Authors searched } 6 \text { databases. } \\
\text { - Trial registries and grey literature were not searched. } \\
\text { - Key words were not provided. } \\
\text { - No restrictions were applied on the language, publication year or status. }\end{array}$ & $\begin{array}{l}\text { Partial Yes: } \\
\text { - Authors searched } 2 \text { databases. } \\
\text { - Trial registries, grey literature and bibliographies were not searched. } \\
\text { - Key words and the search strategy were provided in Fig. 1. } \\
\text { - Restrictions were applied on the language (only articles written in English). }\end{array}$ \\
\hline 5. & $\begin{array}{c}\text { Yes: As stated on page 602:"For each stage, } 2 \text { independent reviewers were } \\
\text { used. If their opinions differed, a third reviewer independently reviewed the } \\
\text { article and decisions, and compared the results". }\end{array}$ & $\begin{array}{c}\text { Yes: As stated on page 3: "Two observers (JE and AD) independently } \\
\text { carried out the screening of publications based on the abstracts of the } \\
\text { retrieved publications". }\end{array}$ \\
\hline 6. & $\begin{array}{l}\text { Yes: As stated on page 602:"Finally, all the included studies were double-extracted } \\
\text { onto data extraction sheets, which were compared. If there was disagreement, } \\
\text { a third team member reviewed the article to resolve the disagreement". }\end{array}$ & $\begin{array}{c}\text { No: The review did not contain an explicit statement that authors } \\
\text { performed data extraction in duplicate. }\end{array}$ \\
\hline 7. & $\begin{array}{c}\text { No: The list of the excluded studies and the reasons for exclusion were not } \\
\text { provided. }\end{array}$ & $\begin{array}{l}\text { Yes: The excluded studies and the reasons for exclusion were provided } \\
\qquad \text { in Table } 2 .\end{array}$ \\
\hline 8. & $\begin{array}{l}\text { Partial Yes: Populations, interventions, comparators, outcomes, and the study } \\
\text { design were described, but not in details. Moreover, the authors' names, the } \\
\text { study setting and follow-up were not addressed. }\end{array}$ & $\begin{array}{c}\text { Partial Yes: Populations, interventions, comparators, outcomes, and the } \\
\text { study design were described, but not in details. Moreover, the study } \\
\text { setting and follow-up were not addressed. }\end{array}$ \\
\hline 9. & No: The risk of bias was not assessed. & No: The risk of bias was not assessed. \\
\hline 10. & No: Funding for primary studies was not reported in the review. & No: Funding for primary studies was not reported in the review. \\
\hline 11. & Meta-analysis was not performed. & $\begin{array}{c}\text { No: Authors did not justify combining the data in meta-analysis and did } \\
\text { not investigate the causes of any heterogeneity. }\end{array}$ \\
\hline 12. & Meta-analysis was not performed. & No: The risk of bias was not assessed. \\
\hline 13. & No: The risk of bias was not assessed. & No: The risk of bias was not assessed. \\
\hline 14. & No: Heterogeneity was not addressed. & No: Heterogeneity was not addressed. \\
\hline 15. & Meta-analysis was not performed. & No: Publication bias was not assessed. \\
\hline 16. & $\begin{array}{l}\text { No: Though authors mentioned their funding sources: "Funded by the Wales } \\
\text { Office of Research and Development Health and Social Care grant No. } \\
\text { R00/2/006", they did not explain how they managed the potential conflict of } \\
\text { interest. }\end{array}$ & No: Neither funding nor conflicting interests were stated in the review. \\
\hline
\end{tabular}

PROSPERO - International Prospective Register of Systematic Reviews; PRISMA - Preferred Reporting Items for Systematic Reviews and Meta-Analyses; DOAJ - Directory of Open Access Journals; PQDT - ProQuest Dissertations and Theses; CCT - controlled clinical trial; ROBINS-I - Risk Of Bias In Non-randomized Studies-of Intervention; NRSI - non-randomized studies of intervention; GRADE - Grading of Recommendations Assessment, Development and Evaluation; APF - acidulated phosphate fluoride; EWLS - enamel white spot lesions; ICTRP - International Clinical Trials Registry Platform; CONSORT - Consolidated Standards of Reporting Trials; NIH - National Institutes of Health; SIGLE - System for Information on Grey Literature in Europe; ISI - Institute for Scientific Information.

Supplementary Table 4. Detailed comparison of the reviews assessing topical fluoride application according to the Jadad decision algorithm

\begin{tabular}{|c|c|c|c|}
\hline No. & Review & Are the included reviews addressing the same question? \\
\hline 1 & Tasios $2019^{15}$ & Question \\
\hline 2 & Sardana $2019^{16}$ & Are topical fluorides effective in the management of OIWSLs? \\
\hline 3 & Sardana $2019^{4}$ & Are topical fluorides effective in the management of OIWSLs? \\
\hline 4 & Nascimento $2016^{13}$ & Are topical fluorides effective in the management of OIWSLs? \\
\hline 5 & Lapenaite $2016^{17}$ & Are topical fluorides effective in the management of OIWSLs? \\
6 & Sonesson $2016^{6}$ & Are topical fluorides effective in the management of OIWSLs? \\
\hline 7 & Chen $2013^{18}$ & Are topical fluorides effective in the management of OIWSLs? \\
\hline 8 & Benson $2013^{8}$ & Are topical fluorides effective in the management of OIWSLs? \\
\hline 9 & Chadwick $2005^{19}$ & Are topical fluorides effective in the management of OIWSLs? \\
\hline 10 & Derks $2004^{14}$ & Are topical fluorides effective in the management of OIWSLs? \\
\hline
\end{tabular}


Continued Supplementary Table 4

\begin{tabular}{|c|c|c|}
\hline \multicolumn{3}{|r|}{ Given that the included reviews were addressing the same question, did the reviews include the same trials? } \\
\hline No. & Review & Studies included \\
\hline 1 & Tasios $2019^{15}$ & 1 - Kumar Jena 2015; 2 - Øgaard 1997; 2001; 3 - Stecksén-Blicks et al. 2007; 4 - Vivaldi-Rodrigues et al. 2006 \\
\hline 2 & Sardana $2019^{16}$ & 1 - Sonesson et al. 2014; 2 - van der Kaaij et al. 2015; 3 - Willmot 2004 \\
\hline 3 & Sardana $2019^{4}$ & $\begin{array}{l}1 \text { - Stecksén-Blicks et al. 2007; } 2 \text { - Jiang et al. 2013; } 3 \text { - Hutto Fretty 2014; } 4 \text { - Kirschneck et al. 2016; } 5 \text { - Du et al. 2012; } \\
6 \text { - Huang et al. 2013; } 7 \text { - He et al. 2016; } 8 \text { - Restrepo et al. 2016; 9 - Singh et al. 2016; } 10 \text { - Bock et al. 2017; } 11 \text { - Ebrahimi et al. } 2017\end{array}$ \\
\hline 4 & Nascimento $2016^{13}$ & 1 - Vivaldi-Rodrigues et al. 2006; 2 - Stecksén-Blicks et al. 2007 \\
\hline 5 & Lapenaite $2016^{17}$ & $\begin{array}{l}1 \text { - Farhadian et al. 2008; } 2 \text { - Huang et al. 2013; } 3 \text { - Richter et al. 2011; } 4 \text { - Al Mulla et al. 2010; } 5 \text { - Du et al. 2012; 6 - Enaia et al. } \\
\text { 2011; } 7 \text { - Baeshen et al. } 2011\end{array}$ \\
\hline 6 & Sonesson $2016^{6}$ & 1 - Agarwal et al. 2013; 2 - Akin et al. 2012; 3 - Du et al. 2012; 4 - Huang et al. 2013 \\
\hline 7 & Chen $2013^{18}$ & 1 - Willmot 2004; 2 - Du et al. 2012; 3 - Baeshen et al. 2011; 4 - Andersson et al. 2007 \\
\hline 8 & Benson $2013^{8}$ & 1 - Luther 200; 2 - Stecksén-Blicks et al. 2007; 3 - Øgaard 2006 \\
\hline 9 & Chadwick $2005^{19}$ & 1 - Hirschfield 1978; 2 - Boyd 1992; 3 - Boyd 1993; 4 - D'Agostino et al. 1988; 5 - Denes 1991; 6 - Alexander 2000 \\
\hline 10 & Derks $2004^{14}$ & 1 - D’Agostino et al. 1988; 2 - Alexander and Ripa 2000 \\
\hline
\end{tabular}

\begin{tabular}{c|c|cc}
\multicolumn{3}{|c}{} & Given that different trials were included, did the included reviews use the same selection criteria? \\
\hline No. & Review & Studies included
\end{tabular}

Population: Human patients of any age, sex, ethnicity, or malocclusion.

Intervention: Any intervention administered at the beginning of treatment with the aim to prevent the development of OIWSLs.

Control: A control/placebo group or other intervention.

1 Tasios $2019^{15}$ Outcome: The incidence and severity of OIWSLS.

Study design: Randomized or quasi-randomized prospective controlled trials, including both parallel and within-person randomized trials. Limitations: No limitations concerning the language, publication year or status were applied.

Population: Patients undergoing multi-bracketed fixed orthodontic treatment or patients who had OIWSLS at the end of multibracketed fixed orthodontic treatment.

Intervention: Self-applied topical fluorides in the form of fluoridated dentifrices, mouth rinses or home-applied gels used by patients.

Control: Standard treatment, placebo control or no intervention control. Standard treatment (or routine treatment) was pre-defined as the use of any fluoride dentifrice containing 1000 / 1055 / 1100 / 1250 ppm of fluoride, as these are the most common concentrations in over-the-counter available dentifrices which have been found to effectively prevent caries in children and adolescents.

2 Sardana $2019^{16}$ Outcome: Studies that evaluated the prevention of OIWSLs during multi-bracketed fixed orthodontic treatment or the reversal of OIWSLS were included. For studies assessing the prevention of OIWSLs, changes in the incidence of OIWSLs during multi-bracketed fixed orthodontic treatment was the primary outcome, and for studies assessing the reversal of OIWSLs after multi-bracketed fixed orthodontic treatment, a reduction in the size of OIWSLS or mean fluorescence values or the prevalence of OIWSLs were the primary outcomes.

Study design: Randomized or quasi-randomized prospective controlled trials.

Limitations: Only articles written in English were included, with no limitations concerning the publication year or status.

Population: Patients undergoing multi-bracketed fixed orthodontic therapy, or patients having 1 or more OIWSLS after the completion of multi-bracketed fixed orthodontic therapy.

Intervention: Professionally applied topical fluorides in the form of gels, foams or varnishes.

3 Sardana $2019^{4}$ Control: Placebo control or no control.

Outcome: Changes in the incidence, a decrease in the prevalence or changes in the size of OIWSLs.

Study design: Randomized or quasi-randomized prospective controlled trials, including only parallel designs.

Limitations: Only articles written in English were included, with no limitations concerning the publication year or status.

Population: Patients using fixed orthodontic appliances.

Intervention: The use of fluoride-containing dental materials.

4 Nascimento $2016^{13}$ Control: A control group not using these materials.

Outcome: Only studies evaluating the risk of OIWSLs in terms of a binary outcome (the presence or absence of lesions) were included. Study design: RCTs, including both parallel and split-mouth designs.

Limitations: No limitations concerning the language, publication year or status were applied.

Population: Patients of any age undergoing treatment with fixed orthodontic appliances.

Intervention: Fluoride-containing products or casein derivatives used throughout appliance therapy or immediately after debonding.

Control: Not provided.

5 Lapenaite $2016^{17}$ Outcome: The prevention of OIWSLs.

Study design: Randomized or quasi-randomized controlled clinical studies.

Limitations: Only articles written in English and published between 2008 and 2013 were included.

Population: Adolescents and young adults ( $<30$ years) with OIWSLs registered and scored within 3 months after the debonding of fixed orthodontic appliances.

Intervention: Any intervention, except laminate veneers, with the aim to reverse OIWSLs or to improve their esthetic appearance.

6 Sonesson $2016^{6} \begin{aligned} & \text { Control: No treatment, placebo or best clinical practice. } \\ & \text { Outcome: The extent, hardness or appearance of OIWSLs with a follow-up period of at least } 8 \text { weeks, as assessed with visual }\end{aligned}$ clinical scores, photographs, caries detection devices, or patient/therapist satisfaction.

Study design: CCTs (randomized or non-randomized) on >20 subjects, including parallel and split-mouth designs.

Limitations: Only articles written in English and published between 2011 and Oct 31, 2015 were included. 


\section{Continued Supplementary Table 4}

\begin{tabular}{l|l|l}
\multicolumn{4}{|c}{} & Given that different trials were included, did the included reviews use the same selection criteria? \\
\hline No. & Review & Studies included
\end{tabular}

Population: Participants who completed fixed orthodontic treatment and had at least 1 clinically visible lesion on the labial enamel surface upon the removal of fixed orthodontic appliances.

Intervention: Remineralizing agents for the treatment of OIWSLs (i.e., any fluoride- or CPP-ACP-based system).

Control: Patients subjected to different agents or not subjected to any intervention (placebo or no intervention).

7 Chen $2013^{18} \quad$ Outcome: Studies in which the primary outcome was a change in the severity of OIWSLs between the experimental and control groups, and the severity was expressed macroscopically in terms of the area over the whiteness of the lesion or microscopically as the amount of mineral loss or the lesion depth.

Study design: RCTs.

Limitations: No limitations concerning the language, publication year or status were applied.

Population: Participants of any age undergoing orthodontic treatment with fixed braces in cases when DWLs were assessed on the teeth remaining in the mouth at the end of orthodontic treatment (at debonding, immediately after the active fixed brace was removed). Intervention: Topical fluorides in the form of toothpastes, mouth rinses, gels, varnishes, or dietary sources at any dose, frequency, duration, or method of administration, and with any of the following active agents/ingredients: NaF, SMFP, SnF $2, A P F$, and AmF.

Control: Individuals not subjected to the fluoride intervention, but instead treated with a placebo, such as a non-fluoride toothpaste and mouth rinse, or given no intervention. Studies involving a control group subjected to an alternative fluoride intervention were also included.

8 Benson $2013^{8}$

Outcome: The presence or absence of new lesions by participant, differences in the size and severity of OIWSLs between the experimental and control groups, any quantitative assessment of enamel mineral loss, such as fluorescent light techniques or microradiography, used with in situ caries models, any participant-assessed outcomes, such as the perception of the lesions and oral health-related quality of life data, or adverse effects.

Study design: RCTs, including only parallel designs.

Limitations: No limitations concerning the language, publication year or status were applied.

Population: Human subjects undergoing orthodontic treatment with fixed appliances.

Intervention: Topical fluorides used throughout appliance therapy.

9 Chadwick $2005^{19}$ Control: Not provided.

Outcome: The outcome measure had to be clearly described and reproducible, i.e., the severity of WSLS or DMFS.

Study design: RCTs, clinical trials, and prospective observational studies with concurrent or historical comparison groups.

Limitations: No limitations concerning the language, publication year or status were applied.

Population: Orthodontic patients in whom demineralization-inhibiting measures were applied during orthodontic treatment. Intervention: Topical fluorides used throughout appliance therapy.

10 Derks $2004^{14}$ Control: Not provided.

Outcome: The preventive measure was used from the beginning of orthodontic treatment with bonded fixed appliances until debonding. Study design: RCTs.

Limitations: Only articles written in English were included, with no limitations concerning the publication year or status.

\begin{tabular}{|c|c|c|c|c|c|}
\hline No. & Review & $\begin{array}{l}\text { Publication } \\
\text { status }\end{array}$ & $\begin{array}{l}\text { Quality and quantity } \\
\text { of primary studies }\end{array}$ & Language restrictions & $\begin{array}{l}\text { Analysis of data } \\
\text { on individual patients }\end{array}$ \\
\hline 1 & Tasios $2019^{15}$ & 2019 & $\begin{array}{l}4 \text { trials: } \\
1 \text { - medium risk; } 3 \text { - high risk }\end{array}$ & no restrictions & aggregate data \\
\hline 2 & Sardana $2019^{16}$ & 2019 & $\begin{array}{c}3 \text { trials: } \\
1 \text { - low risk; } 2 \text { - high risk }\end{array}$ & English-written only & systematic review \\
\hline 3 & Sardana $2019^{4}$ & Epub 2018 & $\begin{array}{l}11 \text { trials: } \\
\text { 5- low risk; } 4 \text { - medium risk; } 2 \text { - high risk }\end{array}$ & English-written only & aggregate data \\
\hline 4 & Nascimento $2016^{13}$ & 2016 & $\begin{array}{l}2 \text { trials: } \\
\text { all trials - high risk }\end{array}$ & no restrictions & aggregate data \\
\hline 5 & Lapenaite $2016^{17}$ & 2016 & $\begin{array}{l}7 \text { trials } \\
\text { quality not assessed }\end{array}$ & English-written only & systematic review \\
\hline 6 & Sonesson $2016^{6}$ & 2016 & $\begin{array}{l}4 \text { trials: } \\
1 \text { - low risk; } 3 \text { - high risk }\end{array}$ & English-written only & systematic review \\
\hline 7 & Chen $2013^{18}$ & 2013 & $\begin{array}{l}4 \text { trials: } \\
1 \text { - medium risk; } 3 \text { - high risk }\end{array}$ & no restrictions & systematic review \\
\hline 8 & Benson $2013^{8}$ & 2013 & $\begin{array}{l}3 \text { trials: } \\
1 \text { - low risk, } 1 \text { - medium risk; } 1 \text { - high risk }\end{array}$ & no restrictions & systematic review \\
\hline 9 & Chadwick $2005^{19}$ & 2005 & $\begin{array}{c}6 \text { trials } \\
\text { quality not assessed }\end{array}$ & no restrictions & systematic review \\
\hline 10 & Derks $2004^{14}$ & 2004 & $\begin{array}{l}2 \text { trials } \\
\text { quality not assessed }\end{array}$ & English-written only & aggregate data \\
\hline
\end{tabular}

OIWSL - orthodontically induced white spot lesion; DWL - deep white lesion; SMFP - sodium monofluorophosphate; AmF - amine fluoride; WSL - white spot lesion; DMFS - decayed, missing, and filled teeth or surfaces. 
Effect of casein phosphopeptide-amorphous calcium phosphate or other casein derivatives in the management of OIWSLs

Four systematic reviews appraised the outcomes from CPP-ACP application: Pithon et al. 2019, ${ }^{20}$ Lapenaite et al. 2016, ${ }^{17}$ Sonesson et al. 2016, ${ }^{6}$ and Chen et al. $2013 .{ }^{18}$ Two out of the 4 reviews found a lack of reliable evidence supporting the effectiveness of CPP-ACP. ${ }^{6,18}$ Considering that these reviews were addressing the same question, using different trials and different selection criteria, the Jadad tool would once again suggest that the best available review be selected according to the superiority in its publication status, the methodological quality of its primary studies, language restrictions, and the analysis of data on individual patients. As a result, Pithon et al. $2019^{20}$ was selected (Fig. 4). The detailed steps of the Jadad decision algorithm are provided in Supplementary Table 5.

Pithon et al. $2019^{20}$ included 11 primary trials (9 RCTs and 2 CCTs) published between 2007 and 2016. The studies used either a parallel or cross-over design. The results indicated that $\mathrm{CPP}$-ACP-containing products were effective in both preventing and reversing OIWSLs.

\section{Effect of fluoride-releasing bonding materials in the management of OIWSLs}

This outcome was addressed by 4 reviews: Tasios et al. 2019, ${ }^{15}$ Nascimento et al. 2016, ${ }^{13}$ Rogers et al. 2010, ${ }^{21}$ and Derks et al. 2004. ${ }^{14}$ The results of these reviews were inconsistent, indicating respectively that fluoridereleasing materials were not effective in the management of OIWSL; fluoride-releasing materials reduced the risk of lesion development; it was impossible to make recommendations on the use of fluoride-containing orthodontic adhesives; and the effect of fluoride-containing bonding materials was not statistically significant.

As in the previous situations, these reviews addressed the same question, but included different trials and utilized different selection criteria. Therefore, the best available review would be selected according to the superiority in its publication status, the methodological quality of its primary studies, language restrictions, and the analysis of data on individual patients. Accordingly, Tasios et al. $2019^{15}$ was selected (Fig. 4). The detailed steps of the Jadad decision algorithm applied from the beginning until the final decision are provided in Supplementary Table 6.

Tasios et al. $2019^{15}$ included 10 studies assessing the effectiveness of fluoride-containing bonding materials in the management of OIWSLs. All the studies had a splitmouth design. Data synthesis revealed that fluoridereleasing adhesives and glass-ionomer bracket adhesives showed no statistically significant differences in comparison with conventional and resin adhesives.

\section{Effect of laser therapy in the management of OIWSLs}

Only 1 systematic review was conducted to evaluate this outcome - Raghis et al. 2018, ${ }^{3}$ including 8 trials (5 RCTs and 3 CCTs) published between 2000 and 2015. Different laser types were utilized in an attempt to manage OIWSLs, including $\mathrm{CO}_{2}$, neodymium-doped yttrium aluminum garnet (Nd:YAG), argon, and Optodan ${ }^{\circledR}$ lasers. All primary studies indicated that laser irradiation was effective in the management of OIWSLs, with the exception of an argon laser with parameters of $250 \mathrm{~mW}, 12 \mathrm{~J} / \mathrm{cm}^{2}$, a beam diameter of $5 \mathrm{~mm}$, and a duration of $10 \mathrm{~s}$. An argon laser within these particular parameters demonstrated a positive effect in a study by Blankenau et al., ${ }^{22}$ whilst no effect was noticed in a study by Elaut and Wehrbein 2004. ${ }^{23}$

\section{Effect of resin infiltration and micro-abrasion in the management of OIWSLs}

The only systematic review to be included which addressed the effect of resin infiltration and micro-abrasion on OIWSLs was by Sonesson et al. 2016. ${ }^{6}$ This review included 2 primary studies ${ }^{24,25}$ that evaluated the efficacy of resin infiltration and 1 primary study ${ }^{26}$ that evaluated the efficacy of micro-abrasion. Although these studies revealed both strategies to be effective, the results of this review indicated a lack of reliable scientific evidence to support camouflaging strategies in managing OIWSLs due to the limited number of available studies.

\section{Discussion}

To the best of our knowledge, this review of reviews represents the first state-of-the-art appraisal of the efficacy of different adjunctive interventions in the management of OIWSLs. The adjunctive interventions in question were topical fluorides, the CPP-ACP products, fluoridereleasing adhesives, laser therapy, resin infiltration, and micro-abrasion. The best available evidence regarding these interventions was derived from 5 separate reviews.

Regarding topical fluoride application, 10 of the systematic reviews made an assessment, 3 of which found a lack of reliable evidence in support of fluoride. Indeed, discordant conclusions are likely due to the conflicting results of primary studies. Therefore, it is worth highlighting 2 gaps in the evidence base in order to interpret these results. Firstly, though many relevant reviews and original studies have been conducted, the best clinical fluoride concentration in the management of OIWSLs was deemed to be unclear; in other words, most primary studies used different fluoride concentrations with different forms of application. A study by Bailey et al. raised warnings against high concentrations of fluoride, which may arrest the remineralization of deeper layers through superficial hypermineralization, thus jeopardizing the esthetic treatment result. ${ }^{27}$ 
Supplementary Table 5. Detailed comparison of the reviews assessing casein phosphopeptide-amorphous calcium phosphate (CPP-ACP) or other casein derivatives according to the Jadad decision algorithm

\begin{tabular}{|c|c|c|}
\multicolumn{2}{c}{} & Are the included reviews addressing the same question? \\
\hline No. & Review & Question \\
\hline 1 & Pithon $2019^{20}$ & Is CPP-ACP application effective in the management of OIWSLs? \\
2 & Lapenaite $2016^{17}$ & Is CPP-ACP application effective in the management of OIWSLs? \\
3 & Sonesson $2016^{6}$ & Is CPP-ACP application effective in the management of OIWSLs? \\
4 & Chen $2013^{18}$ & IS CPP-ACP application effective in the management of OIWSLs?
\end{tabular}

\begin{tabular}{|c|c|c|}
\hline \multicolumn{3}{|r|}{ Given that the included reviews were addressing the same question, did the reviews include the same trials? } \\
\hline No. & Review & Studies included \\
\hline 1 & Pithon $2019^{20}$ & $\begin{array}{l}1 \text { - Akin and Basciftci 2012; } 2 \text { - Andersson et al. 2007; } 3 \text { - Bailey et al. 2009; } 4 \text { - Beerens et al. 2010; } 5 \text { - Bröchner et al. } 2011 \text {; } \\
\text { 6- Heshmat et al. 2014; } 7 \text { - Huang et al. 2013; } 8 \text { - Robertson et al. 2011; } 9 \text { - Singh et al. 2016; } 10 \text { - Uysal et al. 2010; } 11 \text { - Wang et al. } 2012\end{array}$ \\
\hline 2 & Lapenaite $2016^{17}$ & 1 - Huang et al. 2013; 2 - Robertson et al. 2011; 3 - Bailey et al. 2009; 4 - Beerens et al. 2010; 5 - Bröchner et al. 2011 \\
\hline 3 & Sonesson $2016^{6}$ & 1 - Akin and Basciftci 2012; 2 - Huang et al. 2013; 3 - Vashisht 2013 \\
\hline 4 & Chen $2013^{18}$ & 1 - Andersson et al. 2007; 2 - Bröchner et al. 2011; 3 - Bailey et al. 2009; 4 - Beerens et al. 2010 \\
\hline
\end{tabular}

\begin{tabular}{l|l|l}
\multicolumn{5}{|c}{ Given that different trials were included, did the included reviews use the same selection criteria? } \\
\hline No. & Review & Selection criteria
\end{tabular}

Population: Orthodontic patients with labial fixed appliances.

Intervention: The use of CPP-ACP-containing products.

1 Pithon $2019^{20}$ Control: Other oral agents or placebos.

Outcome: The prevention or treatment of OIWSLS around
Study design: CCTs (randomized or non- randomized).

Limitations: No limitations concerning the language, publication year or status were applied.

Population: Patients of any age undergoing treatment with fixed orthodontic appliances.

Intervention: Fluoride-containing products or casein derivatives used throughout appliance therapy or immediately after debonding.

2 Lapenaite $2016^{17}$ Control: Not provided.

Outcome: The prevention of OIWSLs.

Study design: Randomized or quasi-randomized controlled clinical studies.

Limitations: Only articles written in English and published between 2008 and 2013 were included.

Population: Adolescents and young adults ( $<30$ years) with OIWSLs registered and scored within 3 months after the debonding of fixed orthodontic appliances.

Intervention: Any intervention, except laminate veneers, with the aim to reverse OIWSLs or to improve their esthetic appearance.

3 Sonesson 20166 Control: No treatment, placebo or best clinical practice.

Outcome: The extent, hardness or appearance of OIWSLs with a follow-up period of at least 8 weeks, as assessed with visual clinical scores, photographs, caries detection devices, or patient/therapist satisfaction.

Study design: CCTs (randomized or non-randomized) on $>20$ subjects, including parallel and split-mouth designs.

Limitations: Only articles written in English and published between 2011 and Oct. 31, 2015 were included.

Population: Participants who completed fixed orthodontic treatment and had at least 1 clinically visible lesion on the labial enamel surface upon the removal of fixed orthodontic appliances.

Intervention: Remineralizing agents for the treatment of OIWSLs (i.e., any fluoride- or CPP-ACP-based system).

Control: Patients subjected to different agents or not subjected to any intervention (placebo or no intervention).

4 Chen $2013^{18} \quad$ Outcome: Studies in which the primary outcome was a change in the severity of OIWSLs between the experimental and control groups, and the severity was expressed macroscopically in terms of the area over the whiteness of the lesion or microscopically as the amount of mineral loss or the lesion depth.

Study design: RCTs.

Limitations: No limitations concerning the language, publication year or status were applied.

\begin{tabular}{|c|c|c|c|c|}
\hline Review & Publication status & $\begin{array}{l}\text { Quality and quantity } \\
\text { of primary studies }\end{array}$ & Language restrictions & $\begin{array}{c}\text { Analysis of data on individual } \\
\text { patients }\end{array}$ \\
\hline
\end{tabular}

\begin{tabular}{|c|c|c|c|c|c|}
\hline 1 & Pithon $2019^{20}$ & 2019 & $\begin{array}{c}11 \text { trials: } \\
9 \text { - low risk; } 2 \text { - medium risk }\end{array}$ & no restrictions & systematic review \\
\hline 2 & Lapenaite $2016^{17}$ & 2016 & $\begin{array}{l}5 \text { trials } \\
\text { quality not assessed }\end{array}$ & English-written only & systematic review \\
\hline 3 & Sonesson $2016^{6}$ & 2016 & $\begin{array}{c}3 \text { trials: } \\
1 \text { - low risk; } 2 \text { - high risk }\end{array}$ & English-written only & systematic review \\
\hline 4 & Chen $2013^{18}$ & 2013 & $\begin{array}{l}4 \text { trials: } \\
\text { all trials - high risk }\end{array}$ & no restrictions & systematic review \\
\hline
\end{tabular}


Supplementary Table 6. Detailed comparison of the reviews assessing fluoride-containing bonding materials according to the Jadad decision algorithm

\begin{tabular}{|c|c|c|c} 
& \multicolumn{2}{c}{ Are the included reviews addressing the same question? } \\
\hline No. & Review & Question \\
\hline 1 & Tasios $2019^{15}$ & Are fluoride-containing materials effective in the management of OIWSLs? \\
2 & Nascimento $2016^{13}$ & Are fluoride-containing materials effective in the management of OIWSLs? \\
3 & Rogers $2010^{21}$ & Are fluoride-containing materials effective in the management of OIWSLs? \\
4 & Derks $2004^{14}$ & Are fluoride-containing materials effective in the management of OIWSLs?
\end{tabular}

\begin{tabular}{|c|c|c|}
\hline \multicolumn{3}{|r|}{ Given that the included reviews were addressing the same question, did the reviews include the same trials? } \\
\hline No. & Review & Studies included \\
\hline 1 & Tasios $2019^{15}$ & $\begin{array}{c}1 \text { - Allabdullah 2017; } 2 \text { - Banks et al. 1997; } 3 \text { - Gaworski 1999; } 4 \text { - Marcusson et al. 1997; } 5 \text { - Millett et al. 1999; } 6 \text { - Mitchell 1992; } \\
\text { 7 -Trimpeneers and Dermaut 1996; } 8 \text { - Turner 1993; } 9 \text { - Adriaens 1990; } 10 \text { - van der Linden 1998 }\end{array}$ \\
\hline 2 & Nascimento $2016^{13}$ & 1 - Sonis and Snell 1989; 2 - Trimpeneers and Dermaut 1996; 3 - Marcusson et al. 1997; 4 - Millett et al. 2000 \\
\hline 3 & Rogers $2010^{21}$ & $\begin{array}{c}1 \text { - Sonis and Snell 1989; } 2 \text { - Marcusson et al. 1997; } 3 \text { - Millett et al. 1999; } 4 \text { - Gaworski 1999; } 5 \text { - Turner 1993; 6 - Banks et al. 1997; } \\
\text { 7 - Mitchell 1992; } 8 \text { - Trimpeneers and Dermaut 1996; } 9 \text { - Millet et al. 2000; } 10 \text { - Gillgrass } 2001\end{array}$ \\
\hline 4 & Derks $2004^{14}$ & $\begin{array}{c}1 \text { - Mitchell 1992; } 2 \text { - Turner 1993; } 3 \text { - Trimpeneers and Dermaut 1996; } 4 \text { - Marcusson et al. 1997; } 5 \text { - Banks et al. 1997; 6 - Millet et al. 1999; } \\
\text { 7 - Millet et al. } 2000\end{array}$ \\
\hline
\end{tabular}

\begin{tabular}{|c|c|c|}
\hline \multicolumn{3}{|r|}{ Given that different trials were included, did the included reviews use the same selection criteria? } \\
\hline No. & Review & Selection criteria \\
\hline 1 & Tasios $2019^{15}$ & $\begin{array}{l}\text { Population: Human patients of any age, sex, ethnicity, or malocclusion. } \\
\text { Intervention: Any intervention administered at the beginning of treatment with the aim to prevent the development of OIWSLS. } \\
\text { Control: A control/placebo group or other intervention. } \\
\text { Outcome: The incidence and severity of OIWSLs. } \\
\text { Study design: Randomized or quasi-randomized prospective controlled trials, including both parallel and within-person randomized trials. } \\
\text { Limitations: No limitations concerning the language, publication year or status were applied. }\end{array}$ \\
\hline 2 & Nascimento $2016^{13}$ & $\begin{array}{l}\text { Population: Patients using fixed orthodontic appliances. } \\
\text { Intervention: The use of fluoride-containing dental materials. } \\
\text { Control: A control group not using these materials. } \\
\text { Outcome: Only studies evaluating the risk of OIWSLs in terms of a binary outcome (the presence or absence of lesions) were included. } \\
\text { Study design: RCTs, including both parallel and split-mouth designs. } \\
\text { Limitations: No limitations concerning the language, publication year or status were applied. }\end{array}$ \\
\hline 3 & Rogers $2010^{21}$ & $\begin{array}{l}\text { Population: Patients who completed a full course of fixed orthodontic treatment. } \\
\text { Intervention: Fluoride-containing adhesives. } \\
\text { Control: Not provided. } \\
\text { Outcome: Decalcification at the baseline and the end point of the study. When possible, the total number of OIWSLs, } \\
\quad \text { decalcifications as well as DMFT were recorded for the start and finish of the trial for both the subjects and the teeth. } \\
\text { Study design: RCTs, clinical trials, and prospective observational studies with concurrent or historic comparison groups. } \\
\text { Limitations: No limitations concerning the language, publication year or status were applied. }\end{array}$ \\
\hline 4 & Derks $2004^{14}$ & $\begin{array}{l}\text { Population: Orthodontic patients in whom demineralization-inhibiting measures were applied during orthodontic treatment. } \\
\text { Intervention: Topical fluorides used throughout appliance therapy. } \\
\text { Control: Not provided. } \\
\text { Outcome: The preventive measure was used from the beginning of orthodontic treatment with bonded fixed appliances until } \\
\text { debonding. } \\
\text { Study design: RCTs. } \\
\text { Limitations: Only articles written in English were included, with no limitations concerning the publication year or status. }\end{array}$ \\
\hline
\end{tabular}

Given that different selection criteria were used, the Jadad algorithm would suggest assessing and comparing the publication status of primary studies, their methodological quality, language restrictions, and the analysis of data on individual patients.

\begin{tabular}{|c|c|c|c|c|c|}
\hline No. & Review & Publication status & $\begin{array}{l}\text { Quality and quantity } \\
\text { of primary studies }\end{array}$ & Language restrictions & $\begin{array}{l}\text { Analysis of data on individual } \\
\text { patients }\end{array}$ \\
\hline 1 & Tasios $2019^{15}$ & 2019 & $\begin{array}{l}10 \text { trials: } \\
3 \text { - medium risk; } 7 \text { - high risk }\end{array}$ & no restrictions & aggregate data \\
\hline 2 & Nascimento $2016^{13}$ & 2016 & $\begin{array}{l}4 \text { trials: } \\
\text { all trials - high risk }\end{array}$ & $\begin{array}{l}\text { no restrictions; however, only } 1 \\
\text { database searched }\end{array}$ & aggregate data \\
\hline 3 & Rogers $2010^{21}$ & 2010 & $\begin{array}{l}10 \text { trials } \\
\text { quality not assessed }\end{array}$ & no restrictions & systematic review \\
\hline 4 & Derks $2004^{14}$ & 2004 & $\begin{array}{l}7 \text { trials } \\
\text { quality not assessed }\end{array}$ & English-written only & aggregate data \\
\hline
\end{tabular}

DMFT - decayed, missing and filled teeth. 
On the other hand, a study by Sonesson et al. indicated that high concentrations of fluoride could significantly manage OIWSLs. ${ }^{28}$ Secondly, the inappropriate split-mouth design which was used by some primary studies might manipulate the outcomes, as fluorides could be distributed throughout the mouth by saliva and a carry-over or cross-contamination effect would be possible. Consequently, more robust, randomized, parallel-designed CCTs are still required.

As far as CPP-ACP-containing products are concerned, 2 out of 4 reviews found a lack of reliable evidence to support the effectiveness of CPP-ACP. Conflicts could be attributed to the application protocols, which differed with regard to application duration and frequency. ${ }^{27,29}$ The best current evidence, according to the Jadad decision algorithm, indicated that the release of phosphate and calcium ions from the CPP-ACP products led to the saturation of the medium and the re-establishment of oral $\mathrm{pH}$, thereby achieving a remineralization effect. ${ }^{20}$

Concerning the efficacy of fluoride-emitting adhesives, the best available evidence proposed that no statistically significant differences were found between conventional and fluoride-releasing adhesives ${ }^{15}$; however, these results have to be treated with considerable caution, because the primary studies included in all the reviews followed a split-mouth design, which also might result in a crosscontamination effect and incorrect data.

Regarding laser application, the only systematic review which was concerned in this intervention was from Raghis et al. 2018. ${ }^{3}$ Their results proposed that laser therapy was effective in preventing OIWSLs by assuming that laser application would lead to a higher enamel microhardness than in the case of the non-irradiated enamel. ${ }^{3}$

Resin infiltration and micro-abrasion have been recently proposed for camouflaging OIWSLs. Contemporary studies on etching and the development of a material with a high penetration coefficient, called an infiltrant, have expanded the use of the resin infiltration technique. ${ }^{30}$ Regarding technical concerns, the application of resin infiltration must be repeated several times, while the micro-abrasion technique is normally administered at a single appointment. ${ }^{6}$ Although primary studies in the review by Sonesson et al. $2016^{6}$ revealed both techniques to be effective, a definite conclusion could not be drawn due to the limited number of studies. Perhaps more importantly, the long-term success rate of both techniques is still unknown; therefore, these methods should be used selectively on challenging cases.

\section{Limitations}

As with all reviews, this review has strengths and limitations. With regard to strengths, we followed the PRISMA checklist, applied an extensive electronic search, used the AMSTAR-2 tool in order to assess the quality of the selected reviews, and chose the best current evidence from among multiple reviews handling multiple methods of OIWSL management.
The review-of-reviews approach relies on 'secondhand' information, so it is potentially vulnerable to the interpretive biases of the previous reviewers. We attempted to control this using an objective method to choose the best available evidence, i.e., the Jadad decision algorithm. Additionally, all the included reviews ranged in quality between critically low to moderate and some results were ultimately drawn based on critically-low-quality reviews. The heterogeneity of the topical fluoride concentrations used in primary studies, the various CPP-ACP application protocols used and the inappropriate split-mouth design of many of primary studies assessing fluoride-releasing adhesives are considered to be the limitations of this review. Finally, as in all reviews of reviews, the most recent primary trials may not be covered.

\section{Conclusions}

Based on the currently available information and the Jadad decision algorithm criteria, topical fluorides yielded a 25-30\% prevention of OIWSLs; however, their effect on reversing OIWSLs is unclear. The CPP-ACP products are effective in preventing and reversing OIWSLs. Fluoridereleasing adhesives offer no significant differences in comparison with conventional adhesives. Laser irradiation is effective in preventing OIWSLs, with some concerns regarding argon lasers. Finally, there is a lack of a reliable evidence to support resin infiltration and micro-abrasion due to the limited number of available studies.

\section{ORCID iDs}

Wesam Mhd Mounir Bakdach (D) https://orcid.org/0000-0001-6523-0075 Rania Hadad (D) https://orcid.org/0000-0002-8426-143X

\section{References}

1. Ren Y, Jongsma MA, Mei L, van der Mei HC, Busscher HJ. Orthodontic treatment with fixed appliances and biofilm formation - a potential public health threat? Clin Oral Investig. 2014;18(7):1711-1718.

2. Sundararaj D, Venkatachalapathy S, Tandon A, Pereira A. Critical evaluation of incidence and prevalence of white spot lesions during fixed orthodontic appliance treatment: A meta-analysis. $J$ Int Soc Prev Community Dent. 2015;5(6):433-439.

3. Raghis TR, Mahmoud G, Hamadah O. Effectiveness of laser irradiation in preventing enamel demineralization during orthodontic treatment: A systematic review. Dent Med Probl. 2018;55(3):321-332.

4. Sardana D, Zhang J, Ekambaram $M$, Yang $Y$, McGrath $C P$, Yiu CKY. Effectiveness of professional fluorides against enamel white spot lesions during fixed orthodontic treatment: A systematic review and meta-analysis. J Dent. 2019;82:1-10.

5. Sudjalim TR, Woods MG, Manton DJ. Prevention of white spot lesions in orthodontic practice: A contemporary review. Aust Dent J. 2006;51(4):284-289;quiz 347.

6. Sonesson M, Bergstrand F, Gizani S, Twetman S. Management of post-orthodontic white spot lesions: An updated systematic review. Eur J Orthod. 2016;39(2):116-121.

7. Paula ABP, Fernandes AR, Coelho AS, et al. Therapies for white spot lesions - a systematic review. J Evid Based Dent Pract. 2017;17(1):23-38.

8. Benson PE, Parkin N, Dyer F, Millett DT, Furness S, Germain P. Fluorides for the prevention of early tooth decay (demineralised white lesions) during fixed brace treatment. Cochrane Database Syst Rev. 2013;12:CD003809. 
9. Denis M, Atlan A, Vennat E, Tirlet G, Attal JP. White defects on enamel: Diagnosis and anatomopathology: Two essential factors for proper treatment (part 1). Int Orthod. 2013;11(2):139-165.

10. Guzmán-Armstrong S, Chalmers J, Warren JJ. Ask us. White spot lesions: Prevention and treatment. Am J Orthod Dentofacial Orthop. 2010;138(6):690-696.

11. Shea BJ, Reeves BC, Wells G, et al. AMSTAR 2: A critical appraisal tool for systematic reviews that include randomised or non-randomised studies of healthcare interventions, or both. BMJ. 2017;358:j4008.

12. Jadad AR, Cook DJ, Browman GP. A guide to interpreting discordant systematic reviews. CMAJ. 1997;156(10):1411-1416.

13. Nascimento PL de MM, Fernandes MTG, de Figueiredo FED, Faria-ESilva AL. Fluoride-releasing materials to prevent white spot lesions around orthodontic brackets: A systematic review. Braz Dent J. 2016;27(1):101-107.

14. Derks A, Katsaros C, Frencken JE, van't Hof MA, Kuijpers-Jagtman AM. Caries-inhibiting effect of preventive measures during orthodontic treatment with fixed appliances. Caries Res. 2004;38(5):413-420.

15. Tasios T, Papageorgiou SN, Papadopoulos MA, Tsapas A, Haidich A. Prevention of orthodontic enamel demineralization: A systematic review with meta-analyses. Orthod Craniofac Res. 2019;22(4):225-235.

16. Sardana D, Manchanda S, Ekambaram M, Yang $Y$, McGrath $C P$ Yiu CKY. Effectiveness of self-applied topical fluorides against enamel white spot lesions from multi-bracketed fixed orthodontic treatment: A systematic review. Eur J Orthod. 2019;41(6):661-668.

17. Lapenaite $E$, Lopatiene $K$, Ragauskaite A. Prevention and treatment of white spot lesions during and after fixed orthodontic treatment: A systematic literature review. Stomatologija. 2016;18(1):3-8.

18. Chen H, Liu X, Dai J, Jiang Z, Guo T, Ding Y. Effect of remineralizing agents on white spot lesions after orthodontic treatment: A systematic review. Am J Orthod Dentofacial Orthop. 2013;143(3):376-382.e3.

19. Chadwick BL, Roy J, Knox J, Treasure ET. The effect of topical fluorides on decalcification in patients with fixed orthodontic appliances: A systematic review. Am J Orthod Dentofacial Orthop. 2005;128(5):601-606.

20. Pithon MM, Baião FS, Sant'Anna LID, Tanaka OM, Cople-Maia L. Effectiveness of casein phosphopeptide-amorphous calcium phosphate-containing products in the prevention and treatment of white spot lesions in orthodontic patients: A systematic review. J Investig Clin Dent. 2019;10(2):e12391.

21. Rogers S, Chadwick B, Treasure E. Fluoride-containing orthodontic adhesives and decalcification in patients with fixed appliances: A systematic review. Am J Orthod Dentofacial Orthop. 2010;138(4):390.e1-e390.e8.

22. Blankenau RJ, Powell G, Ellis RW, Westerman GH. In vivo caries-like lesion prevention with argon laser: Pilot study. J Clin Laser Med Surg. 1999;17(6):241-243.

23. Elaut $J$, Wehrbein $\mathrm{H}$. The effects of argon laser curing of a resin adhesive on bracket retention and enamel decalcification: A prospective clinical trial. Eur J Orthod. 2004;26(5):553-560.

24. Knösel M, Eckstein A, Helms HJ. Durability of esthetic improvement following Icon resin infiltration of multibracket-induced white spot lesions compared with no therapy over 6 months: A single-center, split-mouth, randomized clinical trial. Am J Orthod Dentofacial Orthop. 2013;144(1):86-96.

25. Senestraro SV., Crowe JJ, Wang M, et al. Minimally invasive resin infiltration of arrested white-spot lesions: A randomized clinical trial. J Am Dent Assoc. 2013;144(9):997-1005.

26. Akin M, Basciftci FA. Can white spot lesions be treated effectively? Angle Orthod. 2012;82(5):770-775.

27. Bailey DL, Adams GG, Tsao CE, et al. Regression of post-orthodontic lesions by a remineralizing cream. J Dent Res. 2009;88(12):1148-1153.

28. Sonesson M, Twetman S, Bondemark L. Effectiveness of highfluoride toothpaste on enamel demineralization during orthodontic treatment - a multicenter randomized controlled trial. Eur J Orthod. 2014;36(6):678-682.

29. Bröchner A, Christensen C, Kristensen B, et al. Treatment of postorthodontic white spot lesions with casein phosphopeptidestabilised amorphous calcium phosphate. Clin Oral Investig. 2011;15(3):369-373.

30. Borges AB, Caneppele TMF, Masterson D, Maia LC. Is resin infiltration an effective esthetic treatment for enamel development defects and white spot lesions? A systematic review. J Dent. 2017;56:11-18. 